\title{
SPECTRAL ESTIMATES FOR SCHRÖDINGER OPERATORS ON PERIODIC DISCRETE GRAPHS
}

\author{
EVGENY KOROTYAEV AND NATALIA SABUROVA
}

\begin{abstract}
We consider normalized Laplacians and their perturbations by periodic potentials (Schrödinger operators) on periodic discrete graphs. The spectrum of the operators consists of an absolutely continuous part, which is a union of a finite number of non-degenerate bands, and a finite number of flat bands, i.e., eigenvalues of infinite multiplicity. We obtain estimates of the Lebesgue measure of the spectrum in terms of geometric parameters of the graphs and show that they become identities for some class of graphs. We determine two-sided estimates on the lengths of the first spectral bands and on the effective masses at the bottom of the spectrum of the Laplace and Schrödinger operators. In particular, these estimates yield that the first spectral band of the Schrödinger operators is non-degenerate.
\end{abstract}

\section{INTRODUCTION}

Laplace operators on graphs have a lot of applications in physics and chemistry, see, e.g., Section 7.6 in [BK13], Chapter 8 in [CDS95], the survey [Ku02] and references therein. There are two types of graphs: discrete and metric. Laplace operators are defined on each of them.

- A discrete graph: the difference Laplacian acts on the space of functions defined on the vertex set of the graph. Here, vertices play the main role, and edges are considered as relations between graph vertices. There are some definitions of the discrete Laplace operators: normalized, combinatorial, weighted Laplacians, see, e.g., [BK13, Ch97, HN09, MW89, S13. The spectrum of the discrete Laplace operator on periodic graphs consists of a finite number of bands separated by gaps.

- A metric graph: the graph is considered as a continuous (metric) space consisting of edges (one-dimensional spaces) connecting graph vertices. Here the Laplacian acts on functions defined along each edge of the graph and satisfying special boundary conditions at the vertices, which guarantee the self-adjointness of the operator in the corresponding $L^{2}$-space, see, e.g., [BK13, KoS99, P12]. The spectrum of the metric Laplace operator on periodic graphs covers the positive semiaxis without an infinite number of gaps.

In the paper [C97] an explicit relation between the spectra of the normalized Laplacian on a discrete graph and its counterpart on the corresponding metric graph with all edges having equal lengths is obtained. Later in the paper [KS15] the eigenfunctions of the continuous spectrum of the Laplacian on a periodic metric graph are expressed in terms of the eigenfunctions of the continuous spectrum of the normalized Laplacian on the corresponding discrete graph. Thus, an explicit relation between the Laplacian on a metric graph and the normalized Laplacian on the corresponding discrete graph is determined. Thereby, the study of the Laplacian spectrum on a metric graph with all edges of equal lengths is reduced to the study of the spectrum of the normalized Laplacian on a discrete graph.

Date: April 9, 2020.

Key words and phrases. discrete Schrödinger operators, periodic graphs, spectral bands. 
In this paper we consider the normalized Laplacian and its perturbations by periodic potentials (Schrödinger operators) on periodic discrete graphs. We do not assume the graph to be embedded into a Euclidean space. But in many applications such a natural embedding exists. For instance, the tight-binding approximation is commonly used to describe the electronic properties of real crystals (see, e.g., [A76]). A crystalline structure is modeled by a discrete graph consisting of vertices representing positions of atoms and edges representing chemical bonds of atoms, by ignoring the physical characters of atoms, which may differ from one another. The model gives good qualitative results in many cases. Under this approach a simple geometric model is a graph embedded into $\mathbb{R}^{d}(d=2,3)$ in such a way that it is invariant with respect to the shifts by integer vectors $m \in \mathbb{Z}^{d}$.

It is known that the spectrum of the Schrödinger operators with periodic potentials on periodic discrete graphs consists of an absolutely continuous part and a finite number of flat bands (i.e., eigenvalues of infinite multiplicity). The absolutely continuous spectrum is a union of a finite number of non-degenerate spectral bands separated by gaps. Here we have a well-known problem: to estimate lengths of the spectral bands and gaps in terms of graph parameters and potentials. In the case of the Schrödinger operator $-\Delta+Q$ with a periodic potential $Q$ in $\mathbb{R}^{d}$ the spectrum is absolutely continuous [T73] and consists of a finite number of non-degenerate spectral bands separated by gaps [Sk87]. There are no flat bands. We note that in the case of $d=1$ there are two-sided estimates of $L^{2}$-norms of potentials in terms of gap lengths, see [K98], [K03], and estimates of the variation of lengths of all bands in terms of $L^{2}$-norms of potentials [K00]. We do not know other estimates.

We describe the main goals of the paper:

1) to obtain two-sided estimates on the lengths of the first spectral bands and on the effective masses at the bottom of the spectrum of the normalized Laplace and Schrödinger operators on periodic discrete graphs, and to prove that the first spectral band of the Schrödinger operators is non-degenerate;

2) to estimate the Lebesgue measure of the spectrum and the sum of gap lengths in terms of geometric parameters of the graphs and potentials;

3) to show that the obtained estimates of the Lebesgue measure of the spectrum are sharp, i.e., there exist periodic graphs for which these estimates become identities;

4) to describe the possible number and positions of flat bands for normalized Laplacians on some classes of periodic graphs; to construct a graph, for which the number of flat bands of the Laplacian is maximal.

The results of this paper were used essentially in [KS16] for obtaining spectral estimates for Laplacians on metric graphs.

1.1. Schrödinger operators on periodic graphs. Let $\Gamma=(V, \mathcal{E})$ be a connected infinite graph, possibly having loops and multiple edges. Here $V$ is the set of its vertices and $\mathcal{E}$ is the set of its unoriented edges. It is convenient to assume that each unoriented edge of the graph corresponds to two oppositely directed edges. We denote the set of all oriented edges of the graph $\Gamma$ by $\mathcal{A}$. An edge starting at a vertex $u \in V$ and ending at a vertex $v \in V$ will be denoted as the ordered pair $(u, v) \in \mathcal{A}$ and is said to be incident to the vertices $u$ and $v$. Vertices $u, v \in V$ will be called adjacent and denoted by $u \sim v$, if $(u, v) \in \mathcal{A}$. The inverse edge of $\mathbf{e}=(u, v) \in \mathcal{A}$ will be denoted by $\underline{\mathbf{e}}=(v, u)$. The degree $\varkappa_{v}$ of the vertex $v \in V$ is the number of all edges in $\mathcal{A}$ starting at $v$. 
Throughout, we consider a locally finite $\mathbb{Z}^{d}$-periodic graph $\Gamma, d \geqslant 2$, i.e., a graph satisfying the following conditions:

1) the graph $\Gamma$ is equipped with a free action of the abelian group $\mathbb{Z}^{d}$;

2) the quotient graph $\Gamma_{*}=\Gamma / \mathbb{Z}^{d}$ is finite.

The quotient graph $\Gamma_{*}=\Gamma / \mathbb{Z}^{d}$ is also called the fundamental graph of the periodic graph $\Gamma$. If $\Gamma$ is embedded into the space $\mathbb{R}^{d}$, then the fundamental graph $\Gamma_{*}$ is a graph on the $d-$ dimensional torus $\mathbb{R}^{d} / \mathbb{Z}^{d}$. The fundamental graph $\Gamma_{*}=\left(V_{*}, \mathcal{E}_{*}\right)$ has the vertex set $V_{*}=V / \mathbb{Z}^{d}$, the set $\mathcal{E}_{*}=\mathcal{E} / \mathbb{Z}^{d}$ of unoriented edges and the set $\mathcal{A}_{*}=\mathcal{A} / \mathbb{Z}^{d}$ of oriented edges.

We consider the Hilbert space $\ell^{2}(V)$ of all square summable functions $f: V \rightarrow \mathbb{C}$ equipped with the norm

$$
\|f\|_{\ell^{2}(V)}^{2}=\sum_{v \in V}|f(v)|^{2}<\infty .
$$

The normalized Laplacian (the Laplace operator) $\Delta$ acting on $\ell^{2}(V)$ is defined by

$$
(\Delta f)(v)=f(v)-\sum_{(v, u) \in \mathcal{A}} \frac{1}{\sqrt{\varkappa_{v} \varkappa_{u}}} f(u), \quad f \in \ell^{2}(V), \quad v \in V,
$$

where $\varkappa_{v}$ is the degree of the vertex $v \in V$. The sum in (1.1) is taken over all edges in $\mathcal{A}$ starting at the vertex $v$. It is known (see [MW89]) that the normalized Laplacian $\Delta$ is a bounded self-adjoint operator on $\ell^{2}(V)$ and its spectrum $\sigma(\Delta)$ is a closed subset of the segment $[0,2]$, containing the point 0 , i.e.,

$$
0 \in \sigma(\Delta) \subset[0,2]
$$

We consider the Schrödinger operator $H=\Delta+Q$ acting on $\ell^{2}(V)$. Suppose that the potential $Q$ is real valued and $\mathbb{Z}^{d}$-periodic, i.e., it satisfies

$$
Q(v+m)=Q(v), \quad \forall(v, m) \in V \times \mathbb{Z}^{d},
$$

where $v+m$ denotes the action of $m \in \mathbb{Z}^{d}$ on $v \in V$.

Remark. There are other definitions of Laplacians on graphs, see [MW89]. For example, the combinatorial Laplacian $\Delta_{c}$ is defined as

$$
\left(\Delta_{c} f\right)(v)=\sum_{(v, u) \in \mathcal{A}}(f(v)-f(u)), \quad f \in \ell^{2}(V), \quad v \in V .
$$

The combinatorial Schrödinger operator $\Delta_{c}+Q$ with a periodic potential $Q$ on periodic graphs was studied in [KS14]. We note that in the case of a graph with all vertices having the same degree $\varkappa_{+}$, the normalized Laplacian $\Delta$ and the combinatorial Laplacian $\Delta_{c}$ (and, consequently, their spectra) are related by the simple identity $\Delta_{c}=\varkappa_{+} \Delta$. However, in the case of an arbitrary graph the spectra of these operators, in spite of many similar properties, may have significant differences. For instance, the absolutely continuous spectrum of the normalized Laplacian $\Delta$ on the simplest periodic graph obtained from the square lattice by adding $N$ vertices on each its edge has the form

$$
\sigma_{a c}(\Delta)=[0,2]
$$

(see Proposition 6.2). In the case of the combinatorial Laplacian $\Delta_{c}$ on the same graph with $N=2$ the absolutely continuous spectrum consists of three spectral bands separated by gaps (see [KS14, p. 600]). 
Results about Laplacians on periodic graphs are used in spectral analysis of the Schrödinger operator with a decaying potential and also for the study of the Laplacians on periodic graphs with various defects. We briefly describe these works. The scattering problem for the Schrödinger operator with a decaying potential on the lattice $\mathbb{Z}^{d}, d>1$, was considered in the papers [BS99, IK12, IM14, Ko10, KM17, RS09, SV01] (see also references therein). The scattering on other graphs was studied in [A12, KS15, KMR18, PR18]. The Schrödinger operator with a potential periodic in some directions and finitely supported in other directions on arbitrary periodic graphs was investigated in [KS17a]. In [AIM16, Ku14, KS17b, SS17] the Laplace and Schrödinger operators on periodic graphs with different defects were considered.

1.2. Edge indices. In order to formulate our results we need to define the notion of the edge index, which was introduced in [KS14. Indices are important to study the spectrum of Laplace and Schrödinger operators on periodic graphs, since fiber operators are expressed in terms of edge indices of the fundamental graph $\Gamma_{*}=\left(V_{*}, \mathcal{E}_{*}\right)$ (see the formula (1.10)).

Let $\nu=\# V_{*}$, where $\# A$ is the number of elements in a set $A$. We fix any $\nu$ vertices of the periodic graph $\Gamma$, which are not $\mathbb{Z}^{d}$-equivalent to one another and denote this vertex set by $V_{0}$. We will call the set $V_{0}$ a fundamental vertex set of the graph $\Gamma$. The set $V_{0}$ can be chosen by different ways. However, it is natural to choose this set in the following way. By Lemma 3.1,i, there exists a subgraph $T=\left(V_{T}, \mathcal{E}_{T}\right)$ of the periodic graph $\Gamma$ satisfying the following conditions:

1) $T$ is a tree, i.e., a connected graph without cycles;

2) the set $V_{T}$ consists of $\nu$ vertices of $\Gamma$ that are not $\mathbb{Z}^{d}$-equivalent to each other.

From now on we assume that the fundamental vertex set $V_{0}$ coincides with the vertex set $V_{T}$ of the tree $T$. We note that the graph $T$ is not unique (see Lemma 3.1,i).

For any vertex $v \in V$ the following unique representation holds true:

$$
v=v_{0}+[v], \quad v_{0} \in V_{0}, \quad[v] \in \mathbb{Z}^{d} .
$$

In other words, each vertex $v$ of the periodic graph $\Gamma$ can be obtained from a vertex $v_{0} \in V_{0}$ by the shift by an integer vector $[v] \in \mathbb{Z}^{d}$. We will call the vector $[v]$ the coordinates of the vertex $v$ with respect to the fundamental vertex set $V_{0}$. For any oriented edge $\mathbf{e}=(u, v) \in \mathcal{A}$ we define the edge index $\tau(\mathbf{e})$ as the integer vector given by

$$
\tau(\mathbf{e})=[v]-[u] \in \mathbb{Z}^{d},
$$

where, due to (1.4), we have

$$
u=u_{0}+[u], \quad v=v_{0}+[v], \quad u_{0}, v_{0} \in V_{0}, \quad[u],[v] \in \mathbb{Z}^{d} .
$$

For example, for the graph $\Gamma$ shown in Fig:1 the index of the edge $\left(v_{3}, v_{1}+a_{1}+a_{2}\right)$ is equal to $(1,1)$ and the edge $\left(v_{1}, v_{4}\right)$ has a zero index. Generally speaking, edge indices depend on the choice of the set $V_{0}$.

We define a surjection $\mathfrak{f}_{\mathcal{A}}: \mathcal{A} \rightarrow \mathcal{A}_{*}=\mathcal{A} / \mathbb{Z}^{d}$, which maps each oriented edge $\mathbf{e} \in \mathcal{A}$ of $\Gamma$ to its equivalence class, i.e., an oriented edge $\mathbf{e}_{*}=\mathfrak{f}_{\mathcal{A}}(\mathbf{e})$ of the fundamental graph $\Gamma_{*}$. For each edge $\mathbf{e}_{*} \in \mathcal{A}_{*}$ we define the edge index $\tau\left(\mathbf{e}_{*}\right)$ in the following way:

$$
\tau\left(\mathbf{e}_{*}\right)=\tau(\mathbf{e}) .
$$

In other words, edge indices of the fundamental graph $\Gamma_{*}$ are induced by indices of the corresponding edges of the periodic graph $\Gamma$. The index of a fundamental graph edge with 


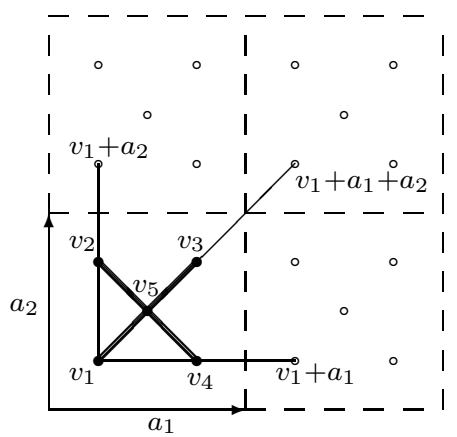

FIGURE 1. A graph $\Gamma$ with the fundamental vertex set $\left\{v_{1}, \ldots, v_{5}\right\}$; only edges of the fundamental graph $\Gamma_{*}$ are shown in the figure; the vectors $a_{1}, a_{2}$ produce an action of the group $\mathbb{Z}^{2}$; edges of the tree $T$ are marked with bold lines.

respect to the fixed fundamental vertex set $V_{0}$ is uniquely determined by formula (1.6), since

$$
\tau(\mathbf{e}+m)=\tau(\mathbf{e}), \quad \forall(\mathbf{e}, m) \in \mathcal{A} \times \mathbb{Z}^{d} .
$$

From the definition (1.5) of the edge index it follows that all edges of the tree $T$ have zero indices, i.e.,

$$
\tau(\mathbf{e})=0, \quad \forall \mathbf{e} \in \mathcal{E}_{T}
$$

Edges with non-zero indices exist on any periodic graph and provide its connectivity. We denote by $\mathcal{B}$ and $\mathcal{B}_{*}$ the sets of all edges with non-zero indices of the periodic graph $\Gamma$ and the fundamental graph $\Gamma_{*}$, respectively.

1.3. The direct integral and the spectrum of the Schrödinger operator. It is well known that periodic operators can be decomposed into a direct integral (for the continuous case see [RS78]). The existence of the direct integral (1.8) for the discrete Schrödinger operator on periodic graphs was discussed in many papers, see [KSS98, Ku89, RR07]. In the case of a concrete periodic graph it is not difficult to write down an explicit expression for the fiber operator (see, e.g., Subsection 4.2.2 in [BK13] and also [HKSW07]). In the case of an arbitrary periodic graph explicit forms of the fiber operators each of which acts in the same space $\ell^{2}\left(V_{*}\right)$, are given in [KS14, KS16a, S13]. Repeating the proof of Theorem 1.1.i for the combinatorial Laplacian in [KS14, we obtain the following statement.

Proposition 1.1. The Schrödinger operator $H=\Delta+Q$ in the space $\ell^{2}(V)$ is decomposed into a constant fiber direct integral

$$
\mathscr{H}=\frac{1}{(2 \pi)^{d}} \int_{\mathbb{T}^{d}}^{\oplus} \ell^{2}\left(V_{*}\right) d \vartheta, \quad U H U^{-1}=\frac{1}{(2 \pi)^{d}} \int_{\mathbb{T}^{d}}^{\oplus} H(\vartheta) d \vartheta,
$$

where $\mathbb{T}^{d}=\mathbb{R}^{d} /(2 \pi \mathbb{Z})^{d}, U: \ell^{2}(V) \rightarrow \mathscr{H}$ is some unitary operator (the Gelfand transformation). Here the fiber Schrödinger operator $H(\vartheta)$ and the fiber Laplacian $\Delta(\vartheta)$ for each $\vartheta \in \mathbb{T}^{d}$ have the form

$$
\begin{gathered}
H(\vartheta)=\Delta(\vartheta)+Q, \\
(\Delta(\vartheta) f)(v)=f(v)-\sum_{\mathbf{e}=(v, u) \in \mathcal{A}_{*}} \frac{e^{i\langle\tau(\mathbf{e}), \vartheta\rangle}}{\sqrt{\varkappa_{v} \varkappa_{u}}} f(u), \quad v \in V_{*},
\end{gathered}
$$


where $\tau(\mathbf{e})$ is the index of the edge $\mathbf{e}$, defined by the formulas (1.5), (1.6); $;\langle\cdot, \cdot\rangle$ is the standard inner product in $\mathbb{R}^{d}$, and $\varkappa_{v}$ is the degree of the vertex $v$.

Remarks. 1) The explicit form (1.10) of the fiber operator $\Delta(\vartheta)$ is important to study spectral properties of Laplace and Schrödinger operators on periodic graphs (see the proofs of Theorems 2.1 2.4).

2) In [S13] the fiber operator $\Delta(\vartheta)$ is expressed in terms of coordinates of edges (considered as directed line segments connecting the corresponding vertices) of the initial periodic graph realized in the space $\mathbb{R}^{d}$. The coordinate vector of each edge

- is a vector in the space $\mathbb{R}^{d}$ (not necessarily integer);

- is a non-zero vector (except for loop edges of the periodic graph).

In contrast to the coordinate vector introduced in [S13], the edge index has the following important properties:

- the edge index is an integer vector in the space $\mathbb{R}^{d}$;

- on the fundamental graph $\Gamma_{*}=\left(V_{*}, \mathcal{E}_{*}\right)$ there exist at least $\# V_{*}-1$ edges with non-zero indices (see the formula (1.7)).

It should be noted that the number of the fundamental graph edges can be arbitrarily large integer number, while the number of edges with non-zero indices can be $d$. For such graphs the dependence of the fiber operator $\Delta(\vartheta)$ on the quasimomentum $\vartheta$ in Sunada's decomposition is rather complicated, while the same dependence in the expression (1.10) is essentially simpler. We also note that in the paper [KS18] we obtained a decomposition of the Laplacian $\Delta$ on periodic graphs into a direct integral where the fiber operator $\Delta(\vartheta)$ depends on the minimal number of non-zero indices and showed that this number is an invariant of the periodic graph.

The decomposition (1.8) and the standard theory of periodic operators (see [RS78], Theorem XIII.85) describe the spectrum of the Schrödinger operator $H=\Delta+Q$. Each fiber operator $H(\vartheta), \vartheta \in \mathbb{T}^{d}$, has $\nu=\# V_{*}$ eigenvalues $\lambda_{n}(\vartheta), n \in \mathbb{N}_{\nu}=\{1, \ldots, \nu\}$, labeled in non-decreasing order counting multiplicity:

$$
\lambda_{1}(\vartheta) \leqslant \lambda_{2}(\vartheta) \leqslant \ldots \leqslant \lambda_{\nu}(\vartheta), \quad \nu=\# V_{*}, \quad \forall \vartheta \in \mathbb{T}^{d} .
$$

Since the operator $H(\vartheta)$ is self-adjoint and analytic in $\vartheta \in \mathbb{T}^{d}$, each $\lambda_{n}(\cdot), n \in \mathbb{N}_{\nu}$, is a realvalued and piecewise analytic function on the torus $\mathbb{T}^{d}$ and defines the spectral band $\sigma_{n}(H)$ :

$$
\sigma_{n}(H):=\sigma_{n}=\left[\lambda_{n}^{-}, \lambda_{n}^{+}\right]=\lambda_{n}\left(\mathbb{T}^{d}\right) .
$$

Then the spectrum of the Schrödinger operator $H$ on the graph $\Gamma$ is given by

$$
\sigma(H)=\bigcup_{\vartheta \in \mathbb{T}^{d}} \sigma(H(\vartheta))=\bigcup_{n=1}^{\nu} \sigma_{n}(H) .
$$

Since the fiber operator $H(\vartheta)$ acts in a finite-dimensional space and is analytic in $\vartheta \in \mathbb{T}^{d}$ (moreover, it is an entire function of $\vartheta \in \mathbb{C}^{d}$ ), according to the general theory, see [GN98, the singular continuous spectrum of the operator $H$ is absent. Note that if $\lambda_{n}(\cdot)=C_{n}=$ const on some subset of $\mathbb{T}^{d}$ of positive Lebesgue measure, then the operator $H$ on the graph $\Gamma$ has the eigenvalue $C_{n}$ of infinite multiplicity. We call $\left\{C_{n}\right\}$ a flat band. Thus, the spectrum of the Schrödinger operator $H$ on the periodic graph $\Gamma$ has the form

$$
\sigma(H)=\sigma_{a c}(H) \cup \sigma_{f b}(H) .
$$


Here $\sigma_{a c}(H)$ is the absolutely continuous spectrum, which is a union of non-degenerate bands from (1.13), and $\sigma_{f b}(H)$ is the set of all eigenvalues of infinite multiplicity (flat bands). An open interval between two neighboring non-degenerate spectral bands is called a gap.

The eigenvalues of the fiber Laplacian $\Delta(\vartheta)$ will be denoted by $\lambda_{n}^{0}(\vartheta), n \in \mathbb{N}_{\nu}$. The spectral bands $\sigma_{n}(\Delta), n \in \mathbb{N}_{\nu}$, for the Laplacian $\Delta$ have the form

$$
\sigma_{n}(\Delta):=\sigma_{n}^{0}=\left[\lambda_{n}^{0-}, \lambda_{n}^{0+}\right]=\lambda_{n}^{0}\left(\mathbb{T}^{d}\right) .
$$

\section{MAin Results}

2.1. Estimates for the first spectral band of the Schrödinger operator. It is known (see [SS92, Theorem 1]) that

$$
\lambda_{1}^{-}=\min _{\vartheta \in \mathbb{T}^{d}} \lambda_{1}(\vartheta)=\lambda_{1}(0) .
$$

The first band function $\lambda_{1}(\vartheta)$ has a Taylor series expansion about the point 0 :

$$
\begin{aligned}
& \lambda_{1}(\vartheta)=\lambda_{1}(0)+\frac{1}{2} \sum_{i, j=1}^{d} M_{i j} \vartheta_{i} \vartheta_{j}+O\left(|\vartheta|^{3}\right), \\
& \text { where } \quad M_{i j}=\frac{\partial^{2} \lambda_{1}(0)}{\partial \vartheta_{i} \partial \vartheta_{j}}, \quad \vartheta=\left(\vartheta_{j}\right)_{j=1}^{d} .
\end{aligned}
$$

The entries of the matrix $m=M^{-1}$, where $M=\left\{M_{i j}\right\}$, represent a tensor, which is called the effective mass tensor at the bottom of the spectrum. The effective mass approximation is a standard approach in solid state physics. By this approach, for energies close to $\lambda_{1}(0)$ a complicated Hamiltonian is replaced by the model Hamiltonian $-\frac{\Delta}{2 m}$, where $\Delta$ is the Laplacian and $m$ is the effective mass. In a general case the effective mass depends on the direction in crystals and represents a tensor.

In the paper [KS16a] the authors obtained upper estimates for the effective masses associated with the ends of each spectral band of the discrete Laplacians on periodic graphs in terms of geometric parameters of the graphs. Moreover, in the case of the bottom of the spectrum they determined two-sided estimates on the effective mass in terms of geometric parameters of the graphs. In the paper [K08] the effective masses for magnetic Schrödinger operators on zigzag nanotubes were estimated. We note that in the case of the Schrödinger operators with periodic potentials in the space $\mathbb{R}^{d}$ the effective mass tensor was studied in [BS04, KiS87, Sh06].

We formulate two-sided estimates on the lengths of the first spectral bands and on the effective masses at the bottom of the spectrum of the normalized Laplace and Schrödinger operators.

Theorem 2.1. i) The smallest eigenvalue $\lambda_{1}(0)$ of the operator $H(0)$ is simple, and all components of a corresponding eigenvector $\psi \in \ell^{2}\left(V_{*}\right)$ are strictly positive.

ii) The first band $\sigma_{1}(H)=\left[\lambda_{1}^{-}, \lambda_{1}^{+}\right]$of the Schrödinger operator $H=\Delta+Q$ is non-degenerate, i.e., $\lambda_{1}^{-}<\lambda_{1}^{+}$, and the following estimate holds:

$$
\begin{aligned}
& c_{0}^{-2}\left|\sigma_{1}(\Delta)\right| \leqslant\left|\sigma_{1}(H)\right| \leqslant c_{0}^{2}\left|\sigma_{1}(\Delta)\right|, \\
& c_{0}=\frac{\psi_{+}}{\psi_{-}}, \quad \psi_{-}=\min _{v \in V_{*}} \frac{\psi(v)}{\sqrt{\varkappa_{v}}}, \quad \psi_{+}=\max _{v \in V_{*}} \frac{\psi(v)}{\sqrt{\varkappa_{v}}},
\end{aligned}
$$

where $\sigma_{1}(\Delta)$ is the first band of the Laplacian $\Delta$, and $\varkappa_{v}$ is the degree of the vertex $v$. 
iii) The effective mass tensors $m_{0}$ and $m$ at the bottom of the spectrum of the Laplacian $\Delta$ and the Schrödinger operator $H=\Delta+Q$, respectively, satisfy:

$$
c_{0}^{-2} m_{0} \leqslant m \leqslant c_{0}^{2} m_{0}
$$

Remarks. 1) Theorem 2.1 remains true for the Schrödinger operator $\Delta_{c}+Q$, where $\Delta_{c}$ is the combinatorial Laplacian defined by (1.3). In this case

$$
\psi_{-}=\min _{v \in V_{*}} \psi(v), \quad \psi_{+}=\max _{v \in V_{*}} \psi(v)
$$

and the proof repeats the proof of Theorem 2.1,

2) For the lattice $\mathbb{Z}^{d}$, Theorem 2.1 was proved in [KiS87]. For an arbitrary periodic graph the estimates (2.3), (2.4) are new.

2.2. Estimate of the Lebesgue measure of the spectrum. We estimate the Lebesgue measure of the spectrum of the Schrödinger operator $H=\Delta+Q$ in terms of geometric parameters of the graph (the number of edges with non-zero indices and vertex degrees) and the sum of gap lengths in terms of geometric parameters of the graph and the potential $Q$.

Theorem 2.2. i) The Lebesgue measure $|\sigma(H)|$ of the spectrum of the Schrödinger operator $H=\Delta+Q$ satisfies the inequality:

$$
|\sigma(H)| \leqslant \sum_{n=1}^{\nu}\left|\sigma_{n}(H)\right| \leqslant 2 \mathfrak{z}, \quad \mathfrak{z}=\sum_{v \in V_{*}} \frac{\mathfrak{z} v}{\varkappa_{v}}
$$

where $\mathfrak{z}_{v}$ is the number of the fundamental graph edges having non-zero indices and starting at the vertex $v \in V_{*}, \varkappa_{v}$ is the degree of the vertex $v$, and $\nu=\# V_{*}$. Moreover, if there exist $s$ gaps $\gamma_{1}(H), \ldots, \gamma_{s}(H)$ in the spectrum of the operator $H$, then the following estimate holds true:

$$
\begin{aligned}
& \sum_{n=1}^{s}\left|\gamma_{n}(H)\right| \geqslant \lambda_{\nu}^{+}-\lambda_{1}^{-}-2 \mathfrak{z} \geqslant C_{0}-2 \mathfrak{z}, \\
& C_{0}=\left|\lambda_{\nu}^{0+}-q_{\bullet}\right|, \quad q_{\bullet}=\max _{v \in V_{*}} Q(v)-\min _{v \in V_{*}} Q(v),
\end{aligned}
$$

where $\lambda_{\nu}^{0+}$ is the upper endpoint of the spectrum of the Laplacian $\Delta$; and $\lambda_{1}^{-}, \lambda_{\nu}^{+}$are the lower and upper endpoints of the spectrum of the Schrödinger operator $H$.

ii) The estimates (2.5) and the first estimate in (2.6) become identities for some classes of graphs, see (2.10).

Remarks. 1) We recall that the spectrum of the normalized Laplacian $\sigma(\Delta) \subset[0,2]$. Therefore, the estimates (2.5) for the Laplacian $\Delta$ are effective if and only if $\mathfrak{z}<1$. This condition holds true when, for each vertex $v \in V_{*}$, the number of edges having non-zero indices and starting at $v$ is sufficiently small compared to the degree of the vertex.

2) The number $\mathfrak{z}$ satisfies the following simple estimates (see Lemma 3.1.ii):

$$
\mathfrak{z} \leqslant 1, \quad \text { if } \quad \nu=1 ; \quad \mathfrak{z} \leqslant \nu-\sum_{v \in V_{*}} \frac{1}{\varkappa_{v}}, \quad \text { if } \quad \nu \geqslant 2 ; \quad \nu=\# V_{*} .
$$


2.3. The Schrödinger operator on loop graphs. A periodic graph $\Gamma$ is called a loop graph if each edge of the fundamental graph

$$
\Gamma_{*}=\left(V_{*}, \mathcal{E}_{*}\right)
$$

with a non-zero index is a loop, i.e., the edge has the form $(v, v)$ for some vertex $v \in V_{*}$.

A loop graph $\Gamma$ is called a precise loop graph if

$$
\cos \left\langle\tau(\mathbf{e}), \vartheta_{0}\right\rangle=-1
$$

for all edges $\mathbf{e} \in \mathcal{B}_{*}$ with non-zero indices and some $\vartheta_{0} \in \mathbb{T}^{d}$, where $\tau(\mathbf{e})$ is the index of the edge $\mathbf{e}$. The point $\vartheta_{0}$ is called a precise quasimomentum of the loop graph $\Gamma$.

Remark. The class of loop graphs is large enough. The simplest example of a precise loop graph is the $d$-dimensional lattice. More complicated examples of loop graphs are discussed in Proposition 2.3 in [KS14.

We describe all spectral bands for the Schrödinger operator on precise loop graphs.

Theorem 2.3. i) Let $\Gamma$ be a loop graph. Then the lower endpoints of the spectral bands $\sigma_{n}=\sigma_{n}(H)=\left[\lambda_{n}^{-}, \lambda_{n}^{+}\right]$of the Schrödinger operator $H$ on the graph $\Gamma$ satisfy

$$
\lambda_{n}^{-}=\lambda_{n}(0), \quad \forall n \in \mathbb{N}_{\nu} .
$$

ii) Let $\Gamma$ be a precise loop graph with a precise quasimomentum $\vartheta_{0} \in \mathbb{T}^{d}$. Then

$$
\begin{aligned}
\sigma_{n}=\left[\lambda_{n}^{-}, \lambda_{n}^{+}\right]= & {\left[\lambda_{n}(0), \lambda_{n}\left(\vartheta_{0}\right)\right], \quad \forall n \in \mathbb{N}_{\nu}, } \\
& \sum_{n=1}^{\nu}\left|\sigma_{n}\right|=2 \mathfrak{z},
\end{aligned}
$$

where $\mathfrak{z}$ is defined in (2.5). In particular, if all edges of the fundamental graph $\Gamma_{*}=\left(V_{*}, \mathcal{E}_{*}\right)$ with non-zero indices have the form $(v, v)$ for some vertex $v \in V_{*}$, then

$$
|\sigma(H)|=\sum_{n=1}^{\nu}\left|\sigma_{n}\right|=2 \mathfrak{z} .
$$

Remarks. 1) By (2.9), the total length of the spectral bands of the Schrödinger operator $H=\Delta+Q$ on precise loop graphs does not depend on the potential $Q$.

2) $\lambda_{n}^{-}, n \in \mathbb{N}_{\nu}$, are the eigenvalues of the Schrödinger operator $H(0)$ defined by the formulas (1.9), (1.10), on the fundamental graph $\Gamma_{*}$. Identities similar to (2.8) hold in the case of $N$ periodic Jacobi matrices on the lattice $\mathbb{Z}$ (and for the Hill operator). The spectrum of these operators is absolutely continuous and is a union of spectral bands separated by gaps. The endpoints of the bands are the so-called $2 N$-periodic eigenvalues.

2.4. Spectrum of the Laplacian on specific periodic graphs. We discuss the possible number of non-degenerate spectral bands and the possible number of eigenvalues of infinite multiplicity (flat bands) for the normalized Laplacian. According to the standard methods of analytic continuation (see, e.g., [RS78, T73]), $\lambda_{*}$ is an eigenvalue of the operator $H$ if and only if $\lambda_{*}=$ const is an eigenvalue of the operator $H(\vartheta)$ for all $\vartheta \in \mathbb{T}^{d}$. We define the multiplicity of a flat band in the following way: a flat band $\left\{\lambda_{*}\right\}$ of the operator $H$ has the multiplicity $m$ if and only if $\lambda_{*}=$ const is an eigenvalue of the operator $H(\vartheta)$ of multiplicity $m$ for almost all $\vartheta \in \mathbb{T}^{d}$. 
Theorem 2.4. Let $\nu, d \geqslant 2$. Then the following statements hold true.

i) There exists a $\mathbb{Z}^{d}$-periodic graph $\Gamma$ such that the spectrum of the Laplacian $\Delta$ on $\Gamma$ consists of two non-degenerate spectral bands and $\nu-2$ flat bands (counting multiplicity) lying in the gap.

ii) There exists a $\mathbb{Z}^{d}$-periodic graph $\Gamma$ such that the spectrum of the operator $\Delta$ on $\Gamma$ has $\left[\frac{\nu-1}{d}\right]$ distinct flat bands, each of which has multiplicity $d-1$ and is embedded into the absolutely continuous spectrum $\sigma_{a c}(\Delta)=[0,2]$.

iii) There exists a $\mathbb{Z}^{d}$-periodic graph $\Gamma$ such that the Lebesgue measure of the spectrum of the Schrödinger operator $H=\Delta+Q$ on $\Gamma$ satisfies $|\sigma(H)|=\frac{4 d}{\nu+2 d-1}$. In particular, we have $|\sigma(H)| \rightarrow 0$ as $\nu \rightarrow \infty$.

Remark. The eigenspace corresponding to an eigenvalue of infinite multiplicity is generated by finitely supported eigenfunctions (see, e.g., [BK13, Theorem 4.5.2]). We note that this statement holds not only in the case of periodic graphs, but also for graphs with a more general structure, see [V05].

We briefly describe the layout of the paper. Auxiliary Section 3 is devoted to a factorization of the fiber Laplacian. In Section 4 we prove Theorems 2.1 and 2.2 about spectral estimates for the Schrödinger operator $H$. In Section 5 we describe the spectrum of the operator $H$ on loop graphs and prove Theorem 2.3. In that section we also study spectral properties of the Laplacian $\Delta$ on bipartite graphs. In Sections 6, 7the spectrum of the Laplace and Schrödinger operators on some perturbations of the $d$-dimensional lattice by adding vertices and edges (in a periodic way) is described. Theorem 2.4 is also proved there. In Section 8 we recall some well-known properties of matrices needed to prove our main results.

\section{Factorization of the Laplace operator}

\subsection{The existence of the tree $T$ and estimates for the number $\mathfrak{z}$.}

Lemma 3.1. i) There exists a subgraph $T=\left(V_{T}, \mathcal{E}_{T}\right)$ of the periodic graph $\Gamma$, satisfying the following conditions:

1) $T$ is a tree, i.e., a connected graph without cycles;

2) the set $V_{T}$ consists of $\nu$ vertices of the graph $\Gamma$, which are not $\mathbb{Z}^{d}$-equivalent to each other.

ii) For the number $\mathfrak{z}$ defined in (2.5), the following estimates hold:

$$
\mathfrak{z} \leqslant 1, \quad \text { if } \quad \nu=1 ; \quad \mathfrak{z} \leqslant \nu-\sum_{v \in V_{*}} \frac{1}{\varkappa_{v}}, \quad \text { if } \quad \nu \geqslant 2 ; \quad \nu=\# V_{*},
$$

where $\varkappa_{v}$ is the degree of the vertex $v$.

Remark. Due to the construction, the graph $T$ is not unique.

Proof. $i)$ We describe a construction of the graph $T=\left(V_{T}, \mathcal{E}_{T}\right)$. First, we put $V_{T}=\varnothing$. Next, let $v$ be an arbitrary vertex of the graph $\Gamma$. We add it to the set $V_{T}:=V_{T} \cup\{v\}$. Since $\Gamma$ is connected, there exist vertices adjacent to $v$. We add to the set $V_{T}$ those of them which are not $\mathbb{Z}^{d}$-equivalent to the vertices from $V_{T}$. We proceed similarly with each vertex newly added to $V_{T}$ and so on. Since the number of the fundamental graph vertices is finite, this process of adding vertices will finish after a finite number of steps. The obtained set $V_{T}$ satisfies the condition 2). Indeed, assume that some vertex $u \in V \backslash V_{T}$ is not $\mathbb{Z}^{d}$-equivalent to any vertex from $V_{T}$. Due to the construction of the set $V_{T}$ and the periodicity of the 
graph $\Gamma$, this means that the vertex $u$ is adjacent neither to vertices from the set $V_{T}$, nor to their equivalent vertices. This contradicts the connectivity of the periodic graph. Thus, the condition 2) holds.

Let $\Gamma_{1}=\left(V_{T}, \mathcal{E}_{1}\right)$ be a subgraph of $\Gamma$ with the vertex set $V_{T}$ and the edge set $\mathcal{E}_{1}$, which contains only edges of $\Gamma$ with both endpoints in $V_{T}$. Due to the construction of the set $V_{T}$, the graph $\Gamma_{1}$ is connected. Then the spanning tree of the graph $\Gamma_{1}$, i.e., a tree containing all vertices of the graph $\Gamma_{1}$, is a required subgraph of $T$.

ii) Let $\nu=1$, i.e., $V_{*}$ consists of a single vertex $v$. Then, by $(2.5), \mathfrak{z}=\frac{\mathfrak{z} v}{\varkappa_{v}} \leqslant 1$.

Now let $\nu \geqslant 2$. Due to the construction of the graph $T$, for each vertex $v$ in the fundamental set $V_{0}=V_{T}$, there exists at least one edge of the tree $T=\left(V_{T}, \mathcal{E}_{T}\right)$ incident to $v$. Then, by (1.6) and (1.7), for each vertex $v \in V_{0}$ and, consequently, for each vertex $v$ of the fundamental graph, the number $\mathfrak{z}_{v}$ of edges having non-zero indices and starting at $v$ is not greater than $\varkappa_{v}-1$. This yields the required estimate:

$$
\mathfrak{z}=\sum_{v \in V_{*}} \frac{\mathfrak{z} v}{\varkappa_{v}} \leqslant \sum_{v \in V_{*}} \frac{\varkappa_{v}-1}{\varkappa_{v}}=\nu-\sum_{v \in V_{*}} \frac{1}{\varkappa_{v}} .
$$

3.2. Factorization of the fiber Laplacian. We introduce the Hilbert space

$$
\ell^{2}(\mathcal{A})=\left\{\phi: \mathcal{A} \rightarrow \mathbb{C} \mid \phi(\underline{\mathbf{e}})=-\phi(\mathbf{e}) \text { for all } \mathbf{e} \in \mathcal{A} \text { and }\langle\phi, \phi\rangle_{\mathcal{A}}<\infty\right\},
$$

with the inner product

$$
\left\langle\phi_{1}, \phi_{2}\right\rangle_{\mathcal{A}}=\frac{1}{2} \sum_{\mathbf{e} \in \mathcal{A}} \phi_{1}(\mathbf{e}) \overline{\phi_{2}(\mathbf{e})} .
$$

It is known (see, e.g., [MW89]), that the Laplacian $\Delta$ has the following factorization:

$$
\Delta=\nabla^{*} \nabla
$$

where the operator $\nabla: \ell^{2}(V) \rightarrow \ell^{2}(\mathcal{A})$ is given by

$$
(\nabla f)(\mathbf{e})=\frac{f(v)}{\sqrt{\varkappa_{v}}}-\frac{f(u)}{\sqrt{\varkappa_{u}}}, \quad f \in \ell^{2}(V), \quad \mathbf{e}=(v, u) \in \mathcal{A} .
$$

The conjugate operator $\nabla^{*}: \ell^{2}(\mathcal{A}) \rightarrow \ell^{2}(V)$ has the form

$$
\left(\nabla^{*} \phi\right)(v)=\frac{1}{\sqrt{\varkappa_{v}}} \sum_{\mathbf{e}=(v, u) \in \mathcal{A}} \phi(\mathbf{e}), \quad \phi \in \ell^{2}(\mathcal{A}), \quad v \in V .
$$

The quadratic form $\langle\Delta f, f\rangle_{V}$ of the Laplacian $\Delta$ is given by

$$
\langle\Delta f, f\rangle_{V}=\frac{1}{2} \sum_{(v, u) \in \mathcal{A}}\left|\frac{f(v)}{\sqrt{\varkappa_{v}}}-\frac{f(u)}{\sqrt{\varkappa_{u}}}\right|^{2},
$$

where $\langle\cdot, \cdot\rangle_{V}$ is the inner product in the space $\ell^{2}(V)$ :

$$
\left\langle f_{1}, f_{2}\right\rangle_{V}=\sum_{v \in V} f_{1}(v) \overline{f_{2}(v)}, \quad \forall f_{1}, f_{2} \in \ell^{2}(V) .
$$

We obtain a similar representation for the fiber Laplacian $\Delta(\vartheta)$. 
Theorem 3.2. i) For each $\vartheta \in \mathbb{T}^{d}$ the fiber Laplacian $\Delta(\vartheta)$ defined by the formula (1.10) satisfies the identity

$$
\Delta(\vartheta)=\nabla^{*}(\vartheta) \nabla(\vartheta)
$$

where the operator $\nabla(\vartheta): \ell^{2}\left(V_{*}\right) \rightarrow \ell^{2}\left(\mathcal{A}_{*}\right)$ is given by

$$
\begin{gathered}
(\nabla(\vartheta) f)(\mathbf{e})=e^{-i\langle\tau(\mathbf{e}), \vartheta\rangle / 2} \frac{f(v)}{\sqrt{\varkappa_{v}}}-e^{i\langle\tau(\mathbf{e}), \vartheta\rangle / 2} \frac{f(u)}{\sqrt{\varkappa_{u}}}, \\
f \in \ell^{2}\left(V_{*}\right), \quad \mathbf{e}=(v, u) \in \mathcal{A}_{*},
\end{gathered}
$$

$\tau(\mathbf{e})$ is the index of the edge $\mathbf{e}$ defined by the formulas (1.5), (1.6). The conjugate operator $\nabla^{*}(\vartheta): \ell^{2}\left(\mathcal{A}_{*}\right) \rightarrow \ell^{2}\left(V_{*}\right)$ has the form

$$
\left(\nabla^{*}(\vartheta) \phi\right)(v)=\frac{1}{\sqrt{\varkappa_{v}}} \sum_{\mathbf{e}=(v, u) \in \mathcal{A}_{*}} e^{i\langle\tau(\mathbf{e}), \vartheta\rangle / 2} \phi(\mathbf{e}), \quad \phi \in \ell^{2}\left(\mathcal{A}_{*}\right), \quad v \in V_{*} .
$$

ii) The quadratic form $\langle\Delta(\vartheta) f, f\rangle_{V_{*}}$ of the fiber Laplacian satisfies the identity

$$
\langle\Delta(\vartheta) f, f\rangle_{V_{*}}=\frac{1}{2} \sum_{\mathbf{e}=(v, u) \in \mathcal{A}_{*}}\left|\frac{f(v)}{\sqrt{\varkappa_{v}}}-e^{i\langle\tau(\mathbf{e}), \vartheta\rangle} \frac{f(u)}{\sqrt{\varkappa_{u}}}\right|^{2},
$$

where summation is over all oriented edges $\mathbf{e} \in \mathcal{A}_{*}$.

Proof. Let $\vartheta \in \mathbb{T}^{d}, f \in \ell^{2}\left(V_{*}\right), \phi \in \ell^{2}\left(\mathcal{A}_{*}\right)$. We denote $\widetilde{f}(v)=\frac{f(v)}{\sqrt{\varkappa_{v}}}$ for all $v \in V_{*}$.

i) First, we prove (3.9) . Using the identity $\tau(\mathbf{e})=-\tau(\underline{\mathbf{e}})$ for all $\mathbf{e} \in \mathcal{A}_{*}$ and the formulas (3.1), (3.2), (3.8), we obtain

$$
\begin{aligned}
&\langle\nabla(\vartheta) f, \phi\rangle_{\mathcal{A}_{*}}=\frac{1}{2} \sum_{\mathbf{e} \in \mathcal{A}_{*}}(\nabla(\vartheta) f)(\mathbf{e}) \overline{\phi(\mathbf{e})} \\
&=\frac{1}{2} \sum_{\mathbf{e}=(v, u) \in \mathcal{A}_{*}}\left(e^{-i\langle\tau(\mathbf{e}), \vartheta\rangle / 2} \widetilde{f}(v)-e^{i\langle\tau(\mathbf{e}), \vartheta\rangle / 2} \widetilde{f}(u)\right) \overline{\phi(\mathbf{e})} \\
&=\frac{1}{2} \sum_{\mathbf{e}=(v, u) \in \mathcal{A}_{*}} e^{-i\langle\tau(\mathbf{e}), \vartheta\rangle / 2} \widetilde{f}(v) \overline{\phi(\mathbf{e})}+\frac{1}{2} \sum_{\underline{\mathbf{e}}=(u, v) \in \mathcal{A}_{*}} e^{-i\langle\tau(\underline{\mathbf{e}}), \vartheta\rangle / 2} \widetilde{f}(u) \overline{\phi(\mathbf{e})} \\
&=\sum_{\mathbf{e}=(v, u) \in \mathcal{A}_{*}} e^{-i\langle\tau(\mathbf{e}), \vartheta\rangle / 2} \widetilde{f}(v) \overline{\phi(\mathbf{e})} .
\end{aligned}
$$

On the other hand, due to (3.6), (3.9), we have

$$
\begin{aligned}
\left\langle f, \nabla^{*}(\vartheta) \phi\right\rangle_{V_{*}} & =\sum_{v \in V_{*}} f(v) \overline{\left(\nabla^{*}(\vartheta) \phi\right)(v)} \\
& =\sum_{v \in V_{*}} \widetilde{f}(v) \sum_{\mathbf{e}=(v, u) \in \mathcal{A}_{*}} e^{-i\langle\tau(\mathbf{e}), \vartheta\rangle / 2} \overline{\phi(\mathbf{e})}=\sum_{\mathbf{e}=(v, u) \in \mathcal{A}_{*}} e^{-i\langle\tau(\mathbf{e}), \vartheta\rangle / 2} \widetilde{f}(v) \overline{\phi(\mathbf{e})} .
\end{aligned}
$$

Comparing (3.11) and (3.12), we see that the operator $\nabla^{*}(\vartheta)$ defined by the formula (3.9) is indeed conjugate of $\nabla(\vartheta)$. 
Next, we prove the identity (3.7). Using (3.8), (3.9) and (1.10), we obtain

$$
\begin{gathered}
\left(\nabla^{*}(\vartheta) \nabla(\vartheta) f\right)(v)=\sum_{\mathbf{e}=(v, u) \in \mathcal{A}_{*}} \frac{1}{\sqrt{\varkappa_{v}}} e^{i\langle\tau(\mathbf{e}), \vartheta\rangle / 2}(\nabla(\vartheta) f)(\mathbf{e}) \\
=\sum_{\mathbf{e}=(v, u) \in \mathcal{A}_{*}} \frac{1}{\sqrt{\varkappa_{v}}} e^{i\langle\tau(\mathbf{e}), \vartheta\rangle / 2}\left(e^{-i\langle\tau(\mathbf{e}), \vartheta\rangle / 2} \widetilde{f}(v)-e^{i\langle\tau(\mathbf{e}), \vartheta\rangle / 2} \widetilde{f}(u)\right) \\
=\sum_{\mathbf{e}=(v, u) \in \mathcal{A}_{*}} \frac{1}{\sqrt{\varkappa_{v}}}\left(\tilde{f}(v)-e^{i\langle\tau(\mathbf{e}), \vartheta\rangle} \tilde{f}(u)\right) \\
=f(v)-\sum_{\mathbf{e}=(v, u) \in \mathcal{A}_{*}} \frac{e^{i\langle\tau(\mathbf{e}), \vartheta\rangle}}{\sqrt{\varkappa_{v} \varkappa_{u}}} f(u)=(\Delta(\vartheta) f)(v), \quad \forall v \in V_{*} .
\end{gathered}
$$

ii) From the identities (3.7), (3.2), (3.8) it follows that

$$
\begin{aligned}
\langle\Delta(\vartheta) f, f\rangle_{V_{*}}=\langle\nabla(\vartheta) f, \nabla(\vartheta) f\rangle_{\mathcal{A}_{*}} & =\frac{1}{2} \sum_{\mathbf{e} \in \mathcal{A}_{*}}|(\nabla(\vartheta) f)(\mathbf{e})|^{2} \\
=\frac{1}{2} \sum_{\mathbf{e}=(v, u) \in \mathcal{A}_{*}}\left|e^{-i\langle\tau(\mathbf{e}), \vartheta\rangle / 2} \widetilde{f}(v)-e^{i\langle\tau(\mathbf{e}), \vartheta\rangle / 2} \widetilde{f}(u)\right|^{2} & =\frac{1}{2} \sum_{\mathbf{e}=(v, u) \in \mathcal{A}_{*}}\left|\widetilde{f}(v)-e^{i\langle\tau(\mathbf{e}), \vartheta\rangle} \widetilde{f}(u)\right|^{2} .
\end{aligned}
$$

\section{Proof of the MAIN RESUlts}

4.1. Estimates for the first spectral band of the Schrödinger operator. We introduce the standard orthonormal basis of the space $\ell^{2}\left(V_{*}\right)$ :

$$
\left(\mathfrak{f}_{u}\right)_{u \in V_{*}}, \quad \text { where } \quad \mathfrak{f}_{u}(v)=\delta_{u v}, \quad v \in V_{*},
$$

$\delta_{u v}$ is the Kronecker delta.

Lemma 4.1. For each $\vartheta \in \mathbb{T}^{d}$ the fiber Laplacian defined by the formula (1.10) in the standard basis (4.1) has the form

$$
\Delta(\vartheta)=\left\{\Delta_{u v}(\vartheta)\right\}_{u, v \in V_{*}}
$$

where

$$
\Delta_{u v}(\vartheta)= \begin{cases}1-\frac{1}{\varkappa_{u}} \sum_{\mathbf{e}=(u, u) \in \mathcal{A}_{*}} \cos \langle\tau(\mathbf{e}), \vartheta\rangle, & \text { if } u=v ; \\ \frac{-1}{\sqrt{\varkappa_{u} \varkappa_{v}}} \sum_{\mathbf{e}=(u, v) \in \mathcal{A}_{*}} e^{-i\langle\tau(\mathbf{e}), \vartheta\rangle,} & \text { if } u \sim v, \quad u \neq v ; \\ 0, & \text { otherwise. }\end{cases}
$$

Here $\varkappa_{v}$ is the degree of the vertex $v, \tau(\mathbf{e})$ is the index of the edge $\mathbf{e}$ defined by the formulas (1.5), (1.6).

Proof. Substituting the formula (1.10) into the identity

$$
\Delta_{u v}(\vartheta)=\left\langle\mathfrak{f}_{u}, \Delta(\vartheta) \mathfrak{f}_{v}\right\rangle_{V_{*}}
$$


and using the fact that for each loop $\mathbf{e}=(u, u) \in \mathcal{A}_{*}$ with the index $\tau(\mathbf{e})$ there exists a loop $\underline{\mathbf{e}}=(u, u) \in \mathcal{A}_{*}$ with the index $-\tau(\mathbf{e})$, and the identity

$$
e^{-i \tau(\mathbf{e})}+e^{i \tau(\mathbf{e})}=2 \cos \tau(\mathbf{e})
$$

we obtain (4.2).

Proof of Theorem 2.1. i) The proof of this item can be found in the paper [SS92]. For the reader's convenience we give these simple arguments. By (4.2), the Laplacian on the fundamental graph $\Gamma_{*}$ in the standard basis (4.1) has the form $\Delta(0)=\left\{\Delta_{u v}(0)\right\}_{u, v \in V_{*}}$, where

$$
\Delta_{u v}(0)=\delta_{u v}-\frac{\varkappa_{u v}}{\sqrt{\varkappa_{u} \varkappa_{v}}}
$$

$\delta_{u v}$ is the Kronecker delta, $\varkappa_{u v}$ is the number of edges of the form $(u, v)$ on the fundamental graph $\Gamma_{*}$, and $\varkappa_{v}$ is the degree of the vertex $v$. Let

$$
A=\mathbb{1}_{\nu}-\Delta(0)
$$

where $\mathbb{1}_{\nu}$ is the identity $(\nu \times \nu)$-matrix, $\nu=\# V_{*}$. By (4.3), all entries of the matrix $A$ are non-negative. Since the fundamental graph $\Gamma_{*}$ is connected, by the matrix property iv), the matrix $A$ is irreducible. The Schrödinger operator on the fundamental graph $\Gamma_{*}$ in the standard basis (4.1) has the form

$$
H(0)=D-A
$$

where

$$
D=\operatorname{diag}(Q(v)+1)_{v \in V_{*}}
$$

is a diagonal matrix. Then, for sufficiently large $t \in \mathbb{R}$,

$$
-H(0)+t \mathbb{1}_{\nu}=A-D+t \mathbb{1}_{\nu}
$$

is an irreducible matrix with non-negative entries. Applying the matrix property $\mathrm{v}$ ) to this matrix, we obtain that the largest eigenvalue of this matrix is simple and there exists a corresponding eigenvector with positive components. This yields the required statement.

To prove the remaining items of Theorem 2.1, we need the following lemma.

Lemma 4.2. Let $\psi \in \ell^{2}\left(V_{*}\right)$ be an eigenvector with positive components corresponding to the smallest eigenvalue $\lambda_{1}(0)$ of the operator $H(0)$. Then

i) for any function $f \in \ell^{2}\left(V_{*}\right)$ and all $\vartheta \in \mathbb{T}^{d}$ the following identity holds

$$
\begin{gathered}
\left\langle\left(H(\vartheta)-\lambda_{1}(0) \mathbb{1}\right) \psi f, \psi f\right\rangle_{V_{*}}=\frac{1}{2} \sum_{\mathbf{e}=(v, u) \in \mathcal{A}_{*}} c_{u v}\left|f(v)-e^{i\langle\tau(\mathbf{e}), \vartheta\rangle} f(u)\right|^{2}, \\
c_{u v}=\frac{\psi(u) \psi(v)}{\sqrt{\varkappa_{u} \varkappa_{v}}},
\end{gathered}
$$

where $\mathbb{1}$ is the identity operator, and $\tau(\mathbf{e})$ is the index of the edge $\mathbf{e} \in \mathcal{A}_{*}$ defined by the formulas (1.5), (1.6);

ii) the following estimate holds:

$$
\begin{aligned}
& c_{0}^{-2} \lambda_{1}^{(0)}(\vartheta) \leqslant \lambda_{1}(\vartheta)-\lambda_{1}(0) \leqslant c_{0}^{2} \lambda_{1}^{(0)}(\vartheta), \quad \forall \vartheta \in \mathbb{T}^{d}, \\
& c_{0}=\frac{\psi_{+}}{\psi_{-}}, \quad \psi_{-}=\min _{v \in V_{*}} \frac{\psi(v)}{\sqrt{\varkappa_{v}}}, \quad \psi_{+}=\max _{v \in V_{*}} \frac{\psi(v)}{\sqrt{\varkappa_{v}}},
\end{aligned}
$$


where $\lambda_{1}(\vartheta)$ and $\lambda_{1}^{(0)}(\vartheta)$ are the smallest eigenvalues of the operators $H(\vartheta)$ and $\Delta(\vartheta)$, respectively.

Proof. i) We use some arguments from [KiS87]. Let $f \in \ell^{2}\left(V_{*}\right)$. From the identities (3.6) and $H(0) \psi=\lambda_{1}(0) \psi$ it follows that

$$
\left\langle\lambda_{1}(0) \psi f, \psi f\right\rangle_{V_{*}}=\sum_{v \in V_{*}} \lambda_{1}(0) \psi^{2}(v)|f(v)|^{2}=\sum_{v \in V_{*}}(H(0) \psi)(v) \psi(v)|f(v)|^{2} .
$$

Then for each $\vartheta \in \mathbb{T}^{d}$, using (1.9), we have

$$
\begin{aligned}
\left\langle\left(H(\vartheta)-\lambda_{1}(0) \mathbb{1}\right) \psi f, \psi f\right\rangle_{V_{*}} & =\sum_{v \in V_{*}}(H(\vartheta) \psi f)(v) \psi(v) \bar{f}(v)-\sum_{v \in V_{*}}(H(0) \psi)(v) \psi(v)|f(v)|^{2} \\
& =\sum_{v \in V_{*}}(\Delta(\vartheta) \psi f)(v) \psi(v) \bar{f}(v)-\sum_{v \in V_{*}}(\Delta(0) \psi)(v) \psi(v)|f(v)|^{2}
\end{aligned}
$$

Substituting the expression (1.10) for the operator $\Delta(\vartheta)$ into the last identity, we obtain

$$
\begin{aligned}
\left\langle\left(H(\vartheta)-\lambda_{1}(0) \mathbb{1}\right) \psi f, \psi f\right\rangle_{V_{*}} & =\sum_{v \in V_{*}}\left(\psi(v) f(v)-\sum_{\mathbf{e}=(v, u) \in \mathcal{A}_{*}} \frac{e^{i\langle\tau(\mathbf{e}), \vartheta\rangle}}{\sqrt{\varkappa_{v} \varkappa_{u}}} \psi(u) f(u)\right) \psi(v) \bar{f}(v) \\
-\sum_{v \in V_{*}}(\psi(v)- & \left.\sum_{\mathbf{e}=(v, u) \in \mathcal{A}_{*}} \frac{\psi(u)}{\sqrt{\varkappa_{v} \varkappa_{u}}}\right) \psi(v)|f(v)|^{2} \\
& =\sum_{\mathbf{e}=(v, u) \in \mathcal{A}_{*}} c_{u v}\left(|f(v)|^{2}-e^{i\langle\tau(\mathbf{e}), \vartheta\rangle} f(u) \bar{f}(v)\right)
\end{aligned}
$$

where $c_{u v}$ is defined in (4.4). By the identity $\tau(\mathbf{e})=-\tau(\underline{\mathbf{e}})$ for all $\mathbf{e} \in \mathcal{A}_{*}$, we have

$$
\begin{aligned}
\sum_{\mathbf{e}=(v, u) \in \mathcal{A}_{*}} c_{u v}\left(|f(v)|^{2}-e^{i\langle\tau(\mathbf{e}), \vartheta\rangle} f(u) \bar{f}(v)\right) & \\
= & \sum_{\underline{\mathbf{e}}=(u, v) \in \mathcal{A}_{*}} c_{u v}\left(|f(v)|^{2}-e^{-i\langle\tau(\underline{\mathbf{e}}), \vartheta\rangle} f(u) \bar{f}(v)\right) \\
& =\sum_{\mathbf{e}=(v, u) \in \mathcal{A}_{*}} c_{u v}\left(|f(u)|^{2}-e^{-i\langle\tau(\mathbf{e}), \vartheta\rangle} f(v) \bar{f}(u)\right) .
\end{aligned}
$$

Then the identity (4.7) can be rewritten in the form:

$$
\begin{aligned}
&\langle(H(\vartheta)\left.\left.-\lambda_{1}(0) \mathbb{1}\right) \psi f, \psi f\right\rangle_{V_{*}} \\
&=\frac{1}{2} \sum_{\mathbf{e}=(v, u) \in \mathcal{A}_{*}} c_{u v}\left(|f(v)|^{2}-e^{i\langle\tau(\mathbf{e}), \vartheta\rangle} f(u) \bar{f}(v)+|f(u)|^{2}-e^{-i\langle\tau(\mathbf{e}), \vartheta\rangle} f(v) \bar{f}(u)\right) \\
&=\frac{1}{2} \sum_{\mathbf{e}=(v, u) \in \mathcal{A}_{*}} c_{u v}\left|f(v)-e^{i\langle\tau(\mathbf{e}), \vartheta\rangle} f(u)\right|^{2}
\end{aligned}
$$

as required.

ii) Let $g=g(\vartheta) \in \ell^{2}\left(V_{*}\right)$ be an eigenvector of the operator

$$
H(\vartheta)-\lambda_{1}(0) \mathbb{1}
$$


corresponding to the smallest eigenvalue $\lambda_{1}(\vartheta)-\lambda_{1}(0)$, such that $\left\|\varkappa^{1 / 2} f_{0}\right\|_{V_{*}}=1$, where $f_{0}=f_{0}(\vartheta)=\psi^{-1} g$, and $\varkappa$ is the operator of multiplication by the function $\varkappa(v)=\varkappa_{v}$. Here $\|\cdot\|_{V_{*}}$ is the norm in the space $\ell^{2}\left(V_{*}\right)$. Then, by the minimax principle (see the matrix property i) and the identities (3.10), (4.4), we have

$$
\begin{aligned}
\lambda_{1}^{(0)}(\vartheta) & =\min _{\substack{f \in \ell^{2}\left(V_{*}\right) \\
\|f\|_{V_{*}=1}}}\langle\Delta(\vartheta) f, f\rangle_{V_{*}} \leqslant\left\langle\Delta(\vartheta) \varkappa^{1 / 2} f_{0}, \varkappa^{1 / 2} f_{0}\right\rangle_{V_{*}} \\
& =\frac{1}{2} \sum_{\mathbf{e}=(v, u) \in \mathcal{A}_{*}}\left|f_{0}(v)-e^{i\langle\tau(\mathbf{e}), \vartheta\rangle} f_{0}(u)\right|^{2} \\
& \leqslant \frac{1}{2 \psi_{-}^{2}} \sum_{\mathbf{e}=(v, u) \in \mathcal{A}_{*}} c_{u v}\left|f_{0}(v)-e^{i\langle\tau(\mathbf{e}), \vartheta\rangle} f_{0}(u)\right|^{2} \\
& =\frac{1}{\psi_{-}^{2}}\left\langle\left(H(\vartheta)-\lambda_{1}(0) \mathbb{1}\right) \psi f_{0}, \psi f_{0}\right\rangle_{V_{*}} \\
& =\frac{\left\|\psi f_{0}\right\|_{V_{*}}^{2}}{\psi_{-}^{2}}\left(\lambda_{1}(\vartheta)-\lambda_{1}(0)\right) \leqslant c_{0}^{2}\left(\lambda_{1}(\vartheta)-\lambda_{1}(0)\right), \quad \forall \vartheta \in \mathbb{T}^{d} .
\end{aligned}
$$

Thus, the first inequality in (4.5) is proved.

Similarly, let $g_{0}=g_{0}(\vartheta) \in \ell^{2}\left(V_{*}\right)$ be an eigenvector of the operator $\Delta(\vartheta)$, corresponding to the smallest eigenvalue $\lambda_{1}^{(0)}(\vartheta)$, such that $\left\|\varkappa^{-1 / 2} f_{0}\right\|_{V_{*}}=1$, where $f_{0}=f_{0}(\vartheta)=\psi g_{0}$. Then

$$
\begin{aligned}
\lambda_{1}(\vartheta)-\lambda_{1}(0)=\min _{\substack{f \in \ell^{2}\left(V_{*}\right) \\
\|f\|_{V_{*}=1}}}\left\langle\left(H(\vartheta)-\lambda_{1}(0) \mathbb{1}\right) f, f\right\rangle_{V_{*}} & \leqslant\left\langle\left(H(\vartheta)-\lambda_{1}(0) \mathbb{1}\right) \varkappa^{-1 / 2} f_{0}, \varkappa^{-1 / 2} f_{0}\right\rangle_{V_{*}} \\
= & \left\langle\left(H(\vartheta)-\lambda_{1}(0) \mathbb{1}\right) \psi \psi^{-1} \varkappa^{-1 / 2} f_{0}, \psi \psi^{-1} \varkappa^{-1 / 2} f_{0}\right\rangle_{V_{*}} \\
= & \frac{1}{2} \sum_{\mathbf{e}=(v, u) \in \mathcal{A}_{*}} c_{u v}\left|\frac{f_{0}(v)}{\psi(v) \sqrt{\varkappa_{v}}}-e^{i\langle\tau(\mathbf{e}), \vartheta\rangle} \frac{f_{0}(u)}{\psi(u) \sqrt{\varkappa_{u}}}\right|^{2} \\
& \leqslant \frac{\psi_{+}^{2}}{2} \sum_{\mathbf{e}=(v, u) \in \mathcal{A}_{*}}\left|\frac{f_{0}(v)}{\psi(v) \sqrt{\varkappa_{v}}}-e^{i\langle\tau(\mathbf{e}), \vartheta\rangle} \frac{f_{0}(u)}{\psi(u) \sqrt{\varkappa_{u}}}\right|^{2} \\
= & \psi_{+}^{2}\left\langle\Delta(\vartheta) \psi^{-1} f_{0}, \psi^{-1} f_{0}\right\rangle_{V_{*}}=\psi_{+}^{2} \lambda_{1}^{(0)}(\vartheta)\left\|\psi^{-1} f_{0}\right\|_{V_{*}}^{2} \leqslant c_{0}^{2} \lambda_{1}^{(0)}(\vartheta), \quad \forall \vartheta \in \mathbb{T}^{d},
\end{aligned}
$$

and the second inequality in (4.5) is proved.

Proof of Theorem 2.1. ii) Using (1.12), (2.1) and (4.5), for some $\vartheta_{+} \in \mathbb{T}^{d}$ we have

$$
\left|\sigma_{1}(H)\right|=\lambda_{1}^{+}-\lambda_{1}(0)=\lambda_{1}\left(\vartheta_{+}\right)-\lambda_{1}(0) \leqslant c_{0}^{2} \lambda_{1}^{(0)}\left(\vartheta_{+}\right) \leqslant c_{0}^{2} \lambda_{1}^{0+}=c_{0}^{2}\left|\sigma_{1}(\Delta)\right| .
$$

Similarly, for some $\vartheta_{+}^{0} \in \mathbb{T}^{d}$ we have

$$
\begin{aligned}
c_{0}^{-2}\left|\sigma_{1}(\Delta)\right|=c_{0}^{-2} \lambda_{1}^{0+} & =c_{0}^{-2} \lambda_{1}^{(0)}\left(\vartheta_{+}^{0}\right) \\
& \leqslant \lambda_{1}\left(\vartheta_{+}^{0}\right)-\lambda_{1}(0) \leqslant \lambda_{1}^{+}-\lambda_{1}(0)=\left|\sigma_{1}(H)\right| .
\end{aligned}
$$

The formulas (2.3) follow from (4.10) and (4.11).

We recall the known fact that the first spectral band $\sigma_{1}(\Delta)=\left[0, \lambda_{1}^{0+}\right]$ of the Laplacian $\Delta$ is non-degenerate. Indeed, assume that 0 is an eigenvalue of the operator $\Delta$ with infinite 
multiplicity. Then (see, e.g., Theorem 4.5.2 in [BK13]) there exists an eigenfunction $0 \neq f \in$ $\ell^{2}(V)$, corresponding to this eigenvalue and having finite support. By (3.5), we obtain

$$
0=\langle\Delta f, f\rangle_{V}=\frac{1}{2} \sum_{(v, u) \in \mathcal{A}}\left|\frac{f(v)}{\sqrt{\varkappa_{v}}}-\frac{f(u)}{\sqrt{\varkappa_{u}}}\right|^{2},
$$

which yields

$$
\frac{f(u)}{\sqrt{\varkappa_{u}}}=\frac{f(v)}{\sqrt{\varkappa_{v}}}, \quad \forall u, v \in V \text { such that } u \sim v .
$$

Since the support of the function $f$ is finite and the graph $\Gamma$ is connected, we conclude that $f=0$. We get a contradiction. Thus, the first spectral band $\sigma_{1}(\Delta)$ of the Laplacian $\Delta$ is non-degenerate. Then, using the fact that $\psi$ is a vector with positive components, from the inequality (2.3) we conclude that the first spectral band $\sigma_{1}(H)$ of the Schrödinger operator $H=\Delta+Q$ is non-degenerate.

iii) The effective mass tensor $m=M^{-1}$, where $M=\left\{M_{i j}\right\}$ is defined in (2.2). Then the estimate (2.4) follows from the inequality (4.5).

4.2. Estimate of the Lebesgue measure of the spectrum. We need the following representation of the fiber Schrödinger operator $H(\vartheta), \vartheta \in \mathbb{T}^{d}$ :

$$
H(\vartheta)=H_{0}+h(\vartheta), \quad H_{0}=\frac{1}{(2 \pi)^{d}} \int_{\mathbb{T}^{d}} H(\vartheta) d \vartheta .
$$

Using the formulas (4.12) and (4.2), we obtain the representation of the operator

$$
h(\vartheta)=\left\{h_{u v}(\vartheta)\right\}_{u, v \in V_{*}}
$$

in the standard basis (4.1):

$$
h_{u v}(\vartheta)=\frac{-1}{\sqrt{\varkappa_{u} \varkappa_{v}}} \sum_{\mathbf{e}=(u, v) \in \mathcal{B}_{*}} e^{-i\langle\tau(\mathbf{e}), \vartheta\rangle},
$$

where $\mathcal{B}_{*}$ is the set of the fundamental graph edges with non-zero indices.

Proof of Theorem 2.2 , i) We define the diagonal matrix $B(\vartheta)$ in the following way:

$$
B(\vartheta)=\operatorname{diag}\left(B_{u}(\vartheta)\right)_{u \in V_{*}}, \quad B_{u}(\vartheta)=\sum_{v \in V_{*}}\left|h_{u v}(\vartheta)\right|, \quad \vartheta \in \mathbb{T}^{d}
$$

From (4.13) it follows that

$$
\left|h_{u v}(\vartheta)\right| \leqslant\left|h_{u v}(0)\right|=\frac{\mathfrak{z}_{u v}}{\sqrt{\varkappa_{u} \varkappa_{v}}}, \quad \forall(u, v, \vartheta) \in V_{*}^{2} \times \mathbb{T}^{d},
$$

where $\mathfrak{z}_{u v}$ is the number of edges of the form $(u, v)$ with non-zero indices on the fundamental graph $\Gamma_{*}$. From (4.14), (4.15) we deduce that

$$
B(\vartheta) \leqslant B(0), \quad \forall \vartheta \in \mathbb{T}^{d} .
$$

The estimate (4.16) and the matrix property vii) give

$$
-B(0) \leqslant-B(\vartheta) \leqslant h(\vartheta) \leqslant B(\vartheta) \leqslant B(0), \quad \forall \vartheta \in \mathbb{T}^{d} .
$$

Combining (4.12) with (4.17), we obtain

$$
H_{0}-B(0) \leqslant H(\vartheta) \leqslant H_{0}+B(0)
$$


whence

$$
\lambda_{n}\left(H_{0}-B(0)\right) \leqslant \lambda_{n}^{-} \leqslant \lambda_{n}(\vartheta) \leqslant \lambda_{n}^{+} \leqslant \lambda_{n}\left(H_{0}+B(0)\right), \quad \forall(n, \vartheta) \in \mathbb{N}_{\nu} \times \mathbb{T}^{d},
$$

which yields

$$
|\sigma(H)| \leqslant \sum_{n=1}^{\nu}\left(\lambda_{n}^{+}-\lambda_{n}^{-}\right) \leqslant \sum_{n=1}^{\nu}\left(\lambda_{n}\left(H_{0}+B(0)\right)-\lambda_{n}\left(H_{0}-B(0)\right)\right)=2 \operatorname{Tr} B(0) .
$$

Using the relations (4.14), (4.15), we have

$$
2 \operatorname{Tr} B(0)=2 \sum_{u \in V_{*}} B_{u}(0)=2 \sum_{u, v \in V_{*}}\left|h_{u v}(0)\right|=2 \sum_{u, v \in V_{*}} \frac{\mathfrak{z}_{u v}}{\sqrt{\varkappa_{u} \varkappa_{v}}} .
$$

By the Cauchy-Schwarz inequality, we obtain

$$
\sum_{u, v \in V_{*}} \frac{\mathfrak{z} u v}{\sqrt{\varkappa_{u} \varkappa_{v}}} \leqslant\left(\sum_{u, v \in V_{*}} \frac{\mathfrak{z} u v}{\varkappa_{u}}\right)^{1 / 2}\left(\sum_{u, v \in V_{*}} \frac{\mathfrak{z} u v}{\varkappa_{v}}\right)^{1 / 2}=\sum_{v \in V_{*}} \frac{\mathfrak{z}_{v}}{\varkappa_{v}} .
$$

Here we have used the identities $\mathfrak{z}_{u v}=\mathfrak{z}_{v u}$ and $\mathfrak{z}_{v}=\sum_{u \in V_{*}} \mathfrak{z}_{v u}$ for all $u, v \in V_{*}$. The estimate (2.5) follows from (4.18)-(4.20).

Now, we prove (2.6) . Since $\lambda_{1}^{-}$and $\lambda_{\nu}^{+}$are the lower and upper endpoints of the spectrum $\sigma(H)$, using the estimate (2.5), we obtain

$$
\sum_{n=1}^{s}\left|\gamma_{n}(H)\right|=\lambda_{\nu}^{+}-\lambda_{1}^{-}-|\sigma(H)| \geqslant \lambda_{\nu}^{+}-\lambda_{1}^{-}-2 \mathfrak{z} .
$$

We rewrite the sequence $(Q(v))_{v \in V_{*}}$ in nondecreasing order

$$
q_{1}^{\bullet} \leqslant q_{2}^{\bullet} \leqslant \ldots \leqslant q_{\nu}^{\bullet}, \quad q_{1}^{\bullet}=0,
$$

where $q_{1}^{\bullet}=Q\left(v_{1}\right), q_{2}^{\bullet}=Q\left(v_{2}\right), \ldots, q_{\nu}^{\bullet}=Q\left(v_{\nu}\right)$ for some distinct vertices $v_{1}, v_{2}, \ldots, v_{\nu} \in V_{*}$, and without loss of generality we may assume that $q_{1}^{\bullet}=0$.

Then, according to the matrix property ii), the eigenvalues of the fiber operator $H(\vartheta)=$ $\Delta(\vartheta)+Q$ satisfy the inequalities

$$
\begin{aligned}
& q_{n}^{\bullet} \leqslant q_{n}^{\bullet}+\lambda_{1}^{0}(\vartheta) \leqslant \lambda_{n}(\vartheta) \leqslant q_{n}^{\bullet}+\lambda_{\nu}^{0}(\vartheta) \leqslant q_{n}^{\bullet}+\lambda_{\nu}^{0+}, \\
& \lambda_{n}^{0}(\vartheta) \leqslant \lambda_{n}(\vartheta) \leqslant \lambda_{n}^{0}(\vartheta)+q_{\nu}^{\bullet}, \quad \forall(\vartheta, n) \in \mathbb{T}^{d} \times \mathbb{N}_{\nu} .
\end{aligned}
$$

From the first inequality in (4.23) we obtain

$$
\lambda_{\nu}^{+} \geqslant q_{\nu}^{\bullet}, \quad \lambda_{1}^{-} \leqslant \lambda_{\nu}^{0+},
$$

and, using the second inequality in (4.23), we have

$$
\begin{gathered}
\lambda_{\nu}^{0+}=\max _{\vartheta \in \mathbb{T}^{d}} \lambda_{\nu}^{0}(\vartheta)=\lambda_{\nu}^{0}\left(\vartheta_{+}\right) \leqslant \lambda_{\nu}\left(\vartheta_{+}\right) \leqslant \lambda_{\nu}^{+}, \\
0=\lambda_{1}^{0-}=\min _{\vartheta \in \mathbb{T}^{d}} \lambda_{1}^{0}(\vartheta)=\lambda_{1}^{0}\left(\vartheta_{-}\right) \geqslant \lambda_{1}\left(\vartheta_{-}\right)-q_{\nu}^{\bullet} \geqslant \lambda_{1}^{-}-q_{\nu}^{\bullet}
\end{gathered}
$$

for some $\vartheta_{-}, \vartheta_{+} \in \mathbb{T}^{d}$. From (4.24)-(4.26) it follows that

$$
\lambda_{\nu}^{+}-\lambda_{1}^{-} \geqslant q_{\nu}^{\bullet}-\lambda_{\nu}^{0+}, \quad \lambda_{\nu}^{+}-\lambda_{1}^{-} \geqslant \lambda_{\nu}^{0+}-q_{\nu}^{\bullet}
$$

which yields $\lambda_{\nu}^{+}-\lambda_{1}^{-} \geqslant C_{0}$, where $C_{0}$ is defined in (2.6). Thus, the estimate (2.6) is proved.

The proof of item ii) will be given in the proof of Theorem 2.3. ii. 


\section{LOOP AND BIPARTITE GRAPHS}

5.1. The Schrödinger operator on loop graphs. Proof of Theorem 2.3 , i) Let $\Gamma$ be a loop graph. Then, by (4.12), (4.13), we obtain

$$
H(\vartheta)=H_{0}+h(\vartheta),
$$

where the operator $h(\vartheta), \vartheta \in \mathbb{T}^{d}$, in the standard basis (4.1) has the form

$$
\begin{aligned}
& h(\vartheta)=\operatorname{diag}\left(h_{v v}(\vartheta)\right)_{v \in V_{*}}, \\
& h_{v v}(\vartheta)=-\frac{1}{\varkappa_{v}} \sum_{\mathbf{e}=(v, v) \in \mathcal{B}_{*}} \cos \langle\tau(\mathbf{e}), \vartheta\rangle,
\end{aligned}
$$

which yields $h_{v v}(0) \leqslant h_{v v}(\vartheta)$ for all $(\vartheta, v) \in \mathbb{T}^{d} \times V_{*}$. Then $h(0) \leqslant h(\vartheta)$ and we have

$$
H(0)=H_{0}+h(0) \leqslant H_{0}+h(\vartheta)=H(\vartheta), \quad \forall \vartheta \in \mathbb{T}^{d},
$$

whence $\lambda_{n}(0) \leqslant \lambda_{n}(\vartheta)$ for all $(\vartheta, n) \in \mathbb{T}^{d} \times \mathbb{N}_{\nu}$, which yields

$$
\lambda_{n}^{-}=\min _{\vartheta \in \mathbb{T}^{d}} \lambda_{n}(\vartheta)=\lambda_{n}(0) .
$$

ii) Let $\Gamma$ be a precise loop graph with a precise quasimomentum $\vartheta_{0} \in \mathbb{T}^{d}$. Then from the identity (5.1) it follows that $h_{v v}(\vartheta) \leqslant h_{v v}\left(\vartheta_{0}\right)$ for all $(\vartheta, v) \in \mathbb{T}^{d} \times V_{*}$, since $\cos \left\langle\tau(\mathbf{e}), \vartheta_{0}\right\rangle=-1$ for all edges $\mathbf{e} \in \mathcal{B}_{*}$ with non-zero indices. Consequently, $h(\vartheta) \leqslant h\left(\vartheta_{0}\right)$ and

$$
H(\vartheta)=H_{0}+h(\vartheta) \leqslant H_{0}+h\left(\vartheta_{0}\right)=H\left(\vartheta_{0}\right),
$$

whence $\lambda_{n}(\vartheta) \leqslant \lambda_{n}\left(\vartheta_{0}\right)$ for all $(\vartheta, n) \in \mathbb{T}^{d} \times \mathbb{N}_{\nu}$. This yields

$$
\lambda_{n}^{+}=\max _{\vartheta \in \mathbb{T}^{d}} \lambda_{n}(\vartheta)=\lambda_{n}\left(\vartheta_{0}\right)
$$

and, by (2.7), the spectral bands $\sigma_{n}$ have the form (2.8).

Using the formulas (2.8) and (5.1), we obtain

$$
\sum_{n=1}^{\nu}\left|\sigma_{n}\right|=\sum_{n=1}^{\nu}\left(\lambda_{n}\left(\vartheta_{0}\right)-\lambda_{n}(0)\right)=\operatorname{Tr}\left(H\left(\vartheta_{0}\right)-H(0)\right)=\operatorname{Tr}\left(h\left(\vartheta_{0}\right)-h(0)\right)=2 \sum_{v \in V_{*}} \frac{\mathfrak{z}_{v}}{\varkappa_{v}},
$$

and the identity (2.9) is proved.

Now, let all edges of the fundamental graph $\Gamma_{*}$ with non-zero indices have the form $(v, v)$ for some vertex $v \in V_{*}$. It only remains to prove the first identity in (2.10). Without loss of generality we may assume that the operator $H(\vartheta)$ in the standard basis (4.1) has the form

$$
H(\vartheta)=\left(\begin{array}{cc}
A & y \\
y^{*} & \Delta_{v v}(\vartheta)+Q(v)
\end{array}\right)
$$

where $y \in \mathbb{C}^{\nu-1}$, the entry $\Delta_{v v}(\vartheta)$ is given by the formula (4.2), and $A$ is a self-adjoint $(\nu-1) \times(\nu-1)$-matrix not depending on $\vartheta$. The eigenvalues $\mu_{1} \leqslant \ldots \leqslant \mu_{\nu-1}$ of the matrix $A$ do not depend on $\vartheta$. Then, applying the matrix property iii), we obtain

$$
\lambda_{1}(\vartheta) \leqslant \mu_{1} \leqslant \lambda_{2}(\vartheta) \leqslant \mu_{2} \leqslant \cdots \leqslant \mu_{\nu-1} \leqslant \lambda_{\nu}(\vartheta), \quad \forall \vartheta \in \mathbb{T}^{d} .
$$

This yields that the spectral bands of the operator $H$ may only touch, but do not overlap, i.e.,

$$
|\sigma(H)|=\sum_{n=1}^{\nu}\left|\sigma_{n}\right|
$$


Thus, in this case the estimate (2.5) and, consequently, the first inequality in (2.6) become identities.

Remark. If there exists $\vartheta_{0} \in \mathbb{T}^{d}$ such that $\left\langle\tau(\mathbf{e}), \vartheta_{0}\right\rangle / \pi$ is odd for all edges $\mathbf{e} \in \mathcal{B}_{*}$ with non-zero indices, then $\vartheta_{0}$ is a precise quasimomentum for $\Gamma$.

5.2. Laplacians on bipartite graphs. A graph is called bipartite if its vertex set is divided into two disjoint sets (called parts of the graph) such that each edge of the graph connects vertices from the distinct parts. Examples of bipartite graphs are the $d$-dimensional lattice and the hexagonal lattice. The face-centered cubic lattice (Fig. [5) is non-bipartite. It is known (see, e.g., [MW89]), that the following statements are equivalent:

- a graph $\Gamma$ is bipartite;

- the point 2 belongs to the spectrum $\sigma(\Delta)$ of the Laplacian $\Delta$ on the graph $\Gamma$;

- the spectrum $\sigma(\Delta)$ is symmetric with respect to the point 1 .

We formulate some spectral properties of the Laplacian on bipartite periodic graphs.

Theorem 5.1. The following statements hold.

i) The fundamental graph $\Gamma_{*}$ is bipartite if and only if for each $\vartheta \in \mathbb{T}^{d}$ the spectrum of the fiber operator $\Delta(\vartheta)$ is symmetric with respect to the point 1 .

ii) Let the fundamental graph $\Gamma_{*}$ be bipartite with parts $V_{1}, V_{2}$ and $m=\# V_{1}-\# V_{2}>0$. Then $\{1\}$ is a flat band of the Laplacian $\Delta$ on the periodic graph $\Gamma$ of multiplicity at least $m$.

iii) If there exist $s$ gaps $\gamma_{1}(\Delta), \ldots, \gamma_{s}(\Delta)$ in the spectrum of the Laplacian $\Delta$ on a bipartite periodic graph $\Gamma$, then the following estimate holds true:

$$
\sum_{n=1}^{s}\left|\gamma_{n}(\Delta)\right| \geqslant 2(1-\mathfrak{z}),
$$

where $\mathfrak{z}$ is defined in (2.5).

iv) Let $\Gamma$ be a bipartite loop graph (the fundamental graph $\Gamma_{*}$ is non-bipartite, since all edges of the graph $\Gamma_{*}$ with non-zero indices are loops). Then each spectral band of the Laplacian $\Delta$ on $\Gamma$ has the form

$$
\sigma_{n}^{0}=\left[\lambda_{n}^{0-}, \lambda_{n}^{0+}\right], \quad n \in \mathbb{N}_{\nu}
$$

where $\lambda_{n}^{0-}$ and $\lambda_{n}^{0+}$ are the eigenvalues of the operators $\Delta(0)$ and $2 \mathbb{1}-\Delta(0)$, respectively (11 is the identity operator).

Proof. $i i)$ Let $\nu_{s}=\# V_{s}, s=1,2$. Since vertices from the same part of the bipartite graph $\Gamma_{*}=\left(V_{*}, \mathcal{E}_{*}\right)$ are not adjacent to each other, for each $\vartheta \in \mathbb{T}^{d}$ the fiber Laplacian (1.10) in the standard basis (4.1) can be represented in the following form:

$$
\Delta(\vartheta)=\left(\begin{array}{cc}
\mathbb{1}_{\nu_{1}} & A(\vartheta) \\
A^{*}(\vartheta) & \mathbb{1}_{\nu_{2}}
\end{array}\right),
$$

where $\mathbb{1}_{n}$ is the identity $(n \times n)$-matrix, and $A(\vartheta)$ is some $\left(\nu_{1} \times \nu_{2}\right)$-matrix. Then $\lambda=1$ is an eigenvalue of the matrix $\Delta(\vartheta)$ with an eigenfunction

$$
f=\left(f_{1}, f_{2}\right) \in \ell^{2}\left(V_{*}\right),
$$

where $f_{s} \in \ell^{2}\left(V_{s}\right)$, if and only if

$$
A(\vartheta) f_{2}=0, \quad A^{*}(\vartheta) f_{1}=0 .
$$


The system $A(\vartheta) f_{2}=0$ always has a zero solution $f_{2}=0$. Since $\operatorname{rank} A^{*}(\vartheta) \leqslant \nu_{2}$, the number of linear independent solutions of the system

$$
A^{*}(\vartheta) f_{1}=0
$$

is equal to

$$
\nu_{1}-\operatorname{rank} A^{*}(\vartheta) \geqslant \nu_{1}-\nu_{2} .
$$

Thus, for each $\vartheta \in \mathbb{T}^{d}$ the operator $\Delta(\vartheta)$ has the eigenvalue $\lambda=1$ of multiplicity at least $\nu_{1}-\nu_{2}$, which yields the required statement.

The proofs of the remaining items repeat the arguments for the combinatorial Laplacian (see [KS14, the proof of Theorem 5.2]).

\section{Perturbations of the $d$-Dimensional Lattice}

We consider $d$-dimensional lattice $\mathbb{L}^{d}=(V, \mathcal{E})$, where the vertex set and the edge set are given by

$$
V=\mathbb{Z}^{d}, \quad \mathcal{E}=\left\{\left(\mathrm{m}, \mathrm{m}+a_{s}\right), \quad \forall \mathrm{m} \in \mathbb{Z}^{d}, \quad s \in \mathbb{N}_{d}\right\},
$$

and the orthonormal basis $a_{1}, \ldots, a_{d}$ coincides with the periods of the lattice $\mathbb{L}^{d}$. The minimal fundamental graph $\mathbb{L}_{*}^{d}$ of the lattice $\mathbb{L}^{d}$ consists of one vertex $v$ and $2 d$ oriented loop edges

$$
\mathbf{e}_{1}=\underline{\mathbf{e}}_{1}=\ldots=\mathbf{e}_{d}=\underline{\mathbf{e}}_{d}=(v, v)
$$

with indices $\pm a_{1}, \ldots, \pm a_{d}$. For $\vartheta_{\pi}=(\pi, \ldots, \pi) \in \mathbb{T}^{d}$ we have the identity

$$
\cos \left\langle\tau(\mathbf{e}), \vartheta_{\pi}\right\rangle=-1
$$

for all edges of the fundamental graph $\mathbb{L}_{*}^{d}$. Consequently, the graph $\mathbb{L}^{d}$ is a precise loop graph with the precise quasimomentum $\vartheta_{\pi}$. It is known that the spectrum of the Laplacian $\Delta$ on $\mathbb{L}^{d}$ has the form

$$
\sigma(\Delta)=\sigma_{a c}(\Delta)=[0,2]
$$

(a)

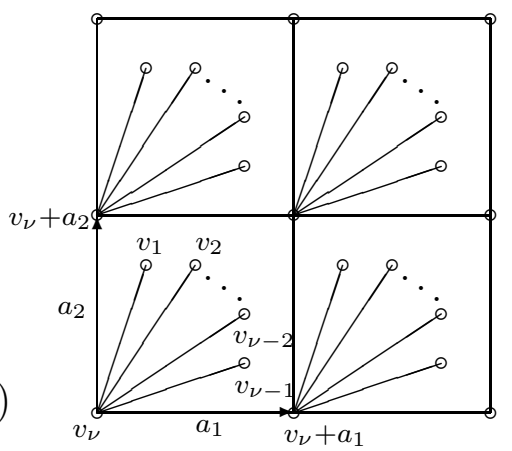

(b)

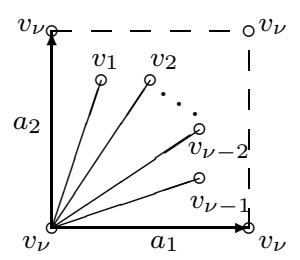

(c)

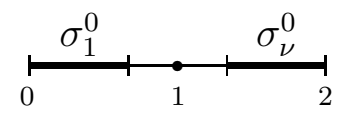

FiguRE 2. a) A $\mathbb{Z}^{d}$-periodic graph $\Gamma$; b) the fundamental graph $\Gamma_{*}$; c) the spectrum of the Laplacian.

We describe the spectrum of the Laplace and Schrödinger operators on perturbations $\Gamma$ of the lattice $\mathbb{L}^{d}$, shown in Fig. 2 $2 a$; these perturbations are precise loop graphs.

Proposition 6.1. Let $\Gamma_{*}$ be obtained from the fundamental graph $\mathbb{L}_{*}^{d}$ of d-dimensional lattice $\mathbb{L}^{d}$ by adding $\nu-1 \geqslant 1$ vertices $v_{1}, \ldots, v_{\nu-1}$ and $\nu-1$ unoriented edges $\left(v_{1}, v_{\nu}\right), \ldots,\left(v_{\nu-1}, v_{\nu}\right)$ with zero indices (see Fig. $2 \mathrm{~b}$ ), $v_{\nu}$ is a unique vertex of $\mathbb{L}_{*}^{d}$. Then $\Gamma$ is a precise loop graph with the precise quasimomentum $\vartheta_{\pi}=(\pi, \ldots, \pi) \in \mathbb{T}^{d}$ and the following statements hold. 
i) The spectrum of the Laplacian $\Delta$ on the graph $\Gamma$ has the form

$$
\sigma(\Delta)=\sigma_{a c}(\Delta) \cup \sigma_{f b}(\Delta), \quad \sigma_{f b}(\Delta)=\{1\},
$$

where the flat band $\{1\}$ has multiplicity $\nu-2$, and the absolutely continuous spectrum $\sigma_{a c}(\Delta)$ consists of two bands $\sigma_{1}^{0}$ and $\sigma_{\nu}^{0}$ :

$$
\sigma_{a c}(\Delta)=\sigma_{1}^{0} \cup \sigma_{\nu}^{0}, \quad \sigma_{1}^{0}=\left[0, \frac{2 d}{\xi}\right], \quad \sigma_{\nu}^{0}=\left[2-\frac{2 d}{\xi}, 2\right], \quad \xi=\nu-1+2 d .
$$

ii) The spectrum of Schrödinger operator $H=\Delta+Q$ on the graph $\Gamma$ has the form

$$
\sigma(H)=\bigcup_{n=1}^{\nu}\left[\lambda_{n}(0), \lambda_{n}\left(\vartheta_{\pi}\right)\right], \quad \vartheta_{\pi}=(\pi, \ldots, \pi) .
$$

iii) Let $q_{j}=Q\left(v_{j}\right), j \in \mathbb{N}_{\nu}$. Without loss of generality we may assume that $q_{\nu}=0$. If all values $q_{1}, \ldots, q_{\nu-1}$ of the potential at the vertices of the fundamental graph $\Gamma_{*}$ are distinct, then $\sigma(H)=\sigma_{a c}(H)$, i.e., $\sigma_{f b}(H)=\varnothing$.

iv) Let among the numbers $q_{1}, \ldots, q_{\nu-1}$ there exist a value $q_{*}$ of multiplicity $m$. Then the spectrum of the Schrödinger operator $H$ on $\Gamma$ has the flat band $\left\{q_{*}+1\right\}$ of multiplicity $m-1$.

$v)$ The Lebesgue measure of the spectrum of the Schrödinger operator $H$ on $\Gamma$ satisfies

$$
|\sigma(H)|=\frac{4 d}{\xi} .
$$

Proof. $i)-i i)$ The fundamental graph $\Gamma_{*}$ consists of $\nu \geqslant 2$ vertices $v_{1}, \ldots, v_{\nu} ; \nu-1$ edges $\left(v_{1}, v_{\nu}\right), \ldots,\left(v_{\nu-1}, v_{\nu}\right)$ with zero indices and $2 d$ oriented loops at the vertex $v_{\nu}$ with the indices $\pm a_{1}, \ldots, \pm a_{d}$. Since all edges of the fundamental graph $\Gamma_{*}$ with non-zero indices are loops and $\cos \left\langle\tau(\mathbf{e}), \vartheta_{\pi}\right\rangle=-1$ for all such edges $\mathbf{e} \in \mathcal{B}_{*}$, the graph $\Gamma$ is a precise loop graph with the precise quasimomentum $\vartheta_{\pi}=(\pi, \ldots, \pi)$. Then, by Theorem 2.3.ii, the spectral bands of the Schrödinger operator $H$ are given by

$$
\sigma_{n}=\left[\lambda_{n}^{-}, \lambda_{n}^{+}\right]=\left[\lambda_{n}(0), \lambda_{n}\left(\vartheta_{\pi}\right)\right], \quad \forall n \in \mathbb{N}_{\nu},
$$

and item $i i)$ is proved.

By (4.2), for each $\vartheta=\left(\vartheta_{j}\right)_{j=1}^{d} \in \mathbb{T}^{d}$ we have

$$
\Delta(\vartheta)=\left(\begin{array}{cccc}
1 & 0 & \ldots & -\frac{1}{\sqrt{\xi}} \\
0 & 1 & \ldots & -\frac{1}{\sqrt{\xi}} \\
\ldots & \ldots & \ldots & \ldots \\
-\frac{1}{\sqrt{\xi}} & -\frac{1}{\sqrt{\xi}} & \ldots & 1-\frac{2 c_{0}}{\xi}
\end{array}\right), \quad\left\{\begin{array}{l}
c_{0}=c_{1}+\ldots+c_{d} \\
c_{j}=\cos \vartheta_{j}, \quad j \in \mathbb{N}_{d} . \\
\xi=\nu-1+2 d
\end{array} .\right.
$$

Using the formula (8.3), we obtain

$$
\begin{aligned}
\operatorname{det}\left(\Delta(0)-\lambda \mathbb{1}_{\nu}\right) & =(1-\lambda)^{\nu-2} \lambda\left(\lambda-2+\frac{2 d}{\xi}\right), \\
\operatorname{det}\left(\Delta\left(\vartheta_{\pi}\right)-\lambda \mathbb{1}_{\nu}\right) & =(1-\lambda)^{\nu-2}(\lambda-2)\left(\lambda-\frac{2 d}{\xi}\right),
\end{aligned}
$$

where $\mathbb{1}_{\nu}$ is the identity $(\nu \times \nu)$-matrix. Then the eigenvalues of the matrices $\Delta(0)$ and $\Delta\left(\vartheta_{\pi}\right)$ have the form

$$
\begin{aligned}
& \lambda_{1}^{0}(0)=0, \quad \lambda_{2}^{0}(0)=\ldots=\lambda_{\nu-1}^{0}(0)=1, \quad \lambda_{\nu}^{0}(0)=2-\frac{2 d}{\xi} \\
& \lambda_{1}^{0}\left(\vartheta_{\pi}\right)=\frac{2 d}{\xi}, \quad \lambda_{2}^{0}\left(\vartheta_{\pi}\right)=\ldots=\lambda_{\nu-1}^{0}\left(\vartheta_{\pi}\right)=1, \quad \lambda_{\nu}^{0}\left(\vartheta_{\pi}\right)=2 .
\end{aligned}
$$


Thus, by (6.4), the spectrum of the Laplacian on the graph $\Gamma$ satisfies the identities (6.2), (6.3).

iii) For each $\vartheta \in \mathbb{T}^{d}$ the operator $H(\vartheta)$ has the form

$$
H(\vartheta)=\Delta(\vartheta)+q, \quad q=\operatorname{diag}\left(q_{1}, \ldots, q_{\nu-1}, 0\right),
$$

where $\Delta(\vartheta)$ is given by (6.6). Using the formula (8.3), we write the characteristic polynomial of the matrix $H(\vartheta)$ in the following form:

$$
\operatorname{det}\left(H(\vartheta)-\lambda \mathbb{1}_{\nu}\right)=\frac{1}{\xi}\left[\left(\xi-\xi \lambda-2 c_{0}\right) W(\lambda)+W^{\prime}(\lambda)\right],
$$

where

$$
W(\lambda)=\left(1+q_{1}-\lambda\right) \ldots\left(1+q_{\nu-1}-\lambda\right) .
$$

We show that $\sigma(H)=\sigma_{a c}(H)$ by contradiction. Suppose that the Schrödinger operator $H$ has an eigenvalue $\lambda$ of infinite multiplicity. Then $\operatorname{det}\left(H(\vartheta)-\lambda \mathbb{1}_{\nu}\right)=0$ for all $\vartheta \in \mathbb{T}^{d}$. The linear combination (6.7) of the linearly independent functions $c_{1}, \ldots, c_{d}, 1$ is identically equal to 0 if and only if

$$
W(\lambda)=0, \quad W^{\prime}(\lambda)=0 .
$$

All values $q_{1}, \ldots, q_{\nu-1}$ of the potential are distinct. Therefore, each zero of the function $W$ is simple. This contradicts the identities (6.9). Consequently, $\sigma(H)=\sigma_{a c}(H)$.

$i v$ ) Without loss of generality we may assume that

$$
q_{1}=q_{2}=\ldots=q_{m}=q_{*} .
$$

Then $\lambda=q_{*}+1$ is a zero of multiplicity $m$ of the function $W$, defined by the formula (6.8), and is a zero of multiplicity $m-1$ of the function $W^{\prime}$. Consequently, the system (6.9), which defines all eigenvalues of infinite multiplicity, has the solution $\lambda=q_{*}+1$ of multiplicity $m-1$. Thus, $\left\{q_{*}+1\right\}$ is a flat band of the operator $H$ with multiplicity $m-1$.

$v$ ) All edges of the fundamental graph $\Gamma_{*}$ with non-zero indices are loops at the vertex $v_{\nu}$ and their number is $2 d$. The degree of this vertex $\varkappa_{\nu}=\xi$. Then the number $\mathfrak{z}$, defined in (2.5), is equal to $\frac{2 d}{\xi}$ and the identity (2.10) takes the form (6.5).

(a)

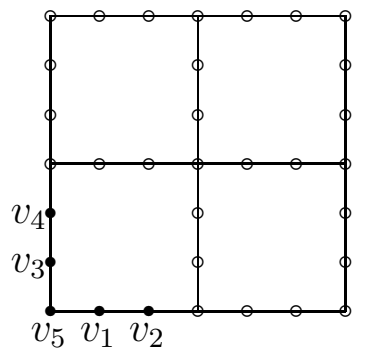

(b)

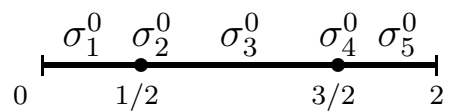

FiguRE 3. a) The graph $\Gamma$ obtained by adding two vertices on each edge of the lattice $\mathbb{L}^{2}$; b) the spectrum of the Laplacian $(d=2, N=2)$.

We describe the spectrum of the Laplacian on the $d$-dimensional lattice with additional vertices (Fig. [3a).

Proposition 6.2. Let $\Gamma$ be the graph obtained from the lattice $\mathbb{L}^{d}$ by adding $N$ vertices on each edge of $\mathbb{L}^{d}$ (for $N=2$ see Fig. 33a). Then the fundamental graph $\Gamma_{*}$ has $\nu=d N+1$ vertices and the spectrum of the Laplacian on $\Gamma$ has the form

$$
\sigma(\Delta)=\sigma_{a c}(\Delta) \cup \sigma_{f b}(\Delta)
$$


where $\sigma_{a c}(\Delta)=[0,2]$ is the absolutely continuous spectrum, and the set of all flat bands is given by:

$$
\sigma_{f b}(\Delta)=\left\{1+\cos \frac{\pi n}{N+1}: n=1, \ldots, N\right\} .
$$

Here each flat band has multiplicity $d-1$ and is embedded into the absolutely continuous spectrum.

Proof. Let $N=1$. The fundamental graph $\Gamma_{*}$ has $\nu=d+1$ vertices. By (4.2), for each

$$
\vartheta=\left(\vartheta_{j}\right)_{j=1}^{d} \in \mathbb{T}^{d}
$$

the operator $\Delta(\vartheta)$ in the standard basis (4.1) has the form

$$
\Delta(\vartheta)=\left(\begin{array}{cccc}
1 & 0 & \ldots & -\frac{1+e^{-i \vartheta_{1}}}{2 \sqrt{d}} \\
0 & 1 & \ldots & -\frac{1+e^{-i \vartheta_{2}}}{2 \sqrt{d}} \\
\ldots & \ldots & \ldots & \ldots \\
-\frac{1+e^{i \vartheta_{1}}}{2 \sqrt{d}} & -\frac{1+e^{i \vartheta_{2}}}{2 \sqrt{d}} & \ldots & 1
\end{array}\right) .
$$

Then the identity $(8.3)$ yields

$$
\begin{aligned}
\operatorname{det}\left(\Delta(\vartheta)-\lambda \mathbb{1}_{\nu}\right) & =(1-\lambda)^{d}\left(1-\lambda-\frac{1}{2 d(1-\lambda)}\left(d+c_{0}\right)\right) \\
& =(1-\lambda)^{d-1}\left(\lambda^{2}-2 \lambda+\frac{d-c_{0}}{2 d}\right),
\end{aligned}
$$

where $c_{0}$ is defined in (6.6). The eigenvalues of the matrix $\Delta(\vartheta)$ have the form

$$
\begin{gathered}
\lambda_{1}^{0}(\vartheta)=1-\sqrt{\frac{d+c_{0}}{2 d}}, \quad \lambda_{d+1}^{0}(\vartheta)=1+\sqrt{\frac{d+c_{0}}{2 d}} ; \\
\lambda_{2}^{0}(\vartheta)=\ldots=\lambda_{d}^{0}(\vartheta)=1 .
\end{gathered}
$$

The identities (6.15) imply that $\{1\}$ is a flat band of the Laplacian $\Delta$ of multiplicity $d-1$. From the identities (6.14) we obtain that

$$
\begin{aligned}
& \lambda_{1}^{0-}=\min _{\vartheta \in \mathbb{T}^{d}} \lambda_{1}^{0}(\vartheta)=\lambda_{1}^{0}(0)=0, \\
& \lambda_{1}^{0+}=\max _{\vartheta \in \mathbb{T}^{d}} \lambda_{1}^{0}(\vartheta)=\lambda_{1}^{0}\left(\vartheta_{\pi}\right)=1, \quad \vartheta_{\pi}=(\pi, \ldots, \pi), \\
& \lambda_{d+1}^{0-}=\min _{\vartheta \in \mathbb{T}^{d}} \lambda_{d+1}^{0}(\vartheta)=\lambda_{d+1}^{0}\left(\vartheta_{\pi}\right)=1, \\
& \lambda_{d+1}^{0+}=\max _{\vartheta \in \mathbb{T}^{d}} \lambda_{d+1}^{0}(\vartheta)=\lambda_{d+1}^{0}(0)=2 .
\end{aligned}
$$

Thus,

$$
\sigma_{1}^{0}=[0,1], \quad \sigma_{d+1}^{0}=[1,2], \quad \sigma_{a c}(\Delta)=\sigma_{1}^{0} \cup \sigma_{d+1}^{0}=[0,2] .
$$

Let $N \geqslant 2$. The fundamental graph $\Gamma_{*}$ has $\nu=d N+1$ vertices. In this case it is more convenient to consider the operator $J=\mathbb{1}-\Delta$ instead of the Laplacian $\Delta$, where $\mathbb{1}$ is the identity operator.

The operator $J(\vartheta)=\mathbb{1}-\Delta(\vartheta)$ in the standard basis (4.1) has the form

$$
J(\vartheta)=\left(\begin{array}{cc}
J_{d N} & y(\vartheta) \\
y^{*}(\vartheta) & 0
\end{array}\right), \quad \forall \vartheta=\left(\vartheta_{j}\right)_{j=1}^{d} \in \mathbb{T}^{d},
$$


where the $(d N \times d N)$-matrix $J_{d N}=\operatorname{diag}\left(J_{N}, \ldots, J_{N}\right)$ and the vector $y(\vartheta)$ are given by

$$
\begin{aligned}
& J_{N}=\frac{1}{2}\left(\begin{array}{cccc}
0 & 1 & 0 & \ldots \\
1 & 0 & 1 & \ldots \\
0 & 1 & 0 & \ldots \\
\cdots & \ldots & \ldots & \ldots
\end{array}\right), \quad y(\vartheta)=\left(\begin{array}{c}
y_{1}\left(\vartheta_{1}\right) \\
\vdots \\
y_{d}\left(\vartheta_{d}\right)
\end{array}\right), \\
& y_{j}\left(\vartheta_{j}\right)=\frac{1}{2 \sqrt{d}}\left(\begin{array}{c}
1 \\
0 \\
\vdots \\
0 \\
e^{-i \vartheta_{j}}
\end{array}\right) \in \mathbb{C}^{N}, \quad j \in \mathbb{N}_{d} .
\end{aligned}
$$

It is known that the eigenvalues of the Jacobi $(N \times N)$-matrix $J_{N}$ are distinct and have the form

$$
\mu_{n}=\cos \frac{\pi n}{N+1} \in(-1,1), \quad n=1, \ldots, N .
$$

Consequently, the matrix $J_{d N}$ has $N$ distinct eigenvalues $\mu_{n}$ of multiplicity $d$. Then, by the matrix property iii), the operator $J$ has at least $N=\frac{\nu-1}{d}$ flat bands $\left\{\mu_{n}\right\}, n \in \mathbb{N}_{N}$, each of which has multiplicity $d-1$.

We describe $\sigma_{a c}(J)$. The identity (8.3) yields

$$
\operatorname{det}\left(\lambda \mathbb{1}_{\nu}-J(\vartheta)\right)=\left(\lambda-y^{*}(\vartheta)\left(\lambda \mathbb{1}_{d N}-J_{d N}\right)^{-1} y(\vartheta)\right) \operatorname{det}\left(\lambda \mathbb{1}_{d N}-J_{d N}\right) .
$$

From the explicit form of the matrix $J_{d N}$ it follows that

$$
\operatorname{det}\left(\lambda \mathbb{1}_{d N}-J_{d N}\right)=\mathcal{D}_{N}^{d}(\lambda), \quad \text { where } \mathcal{D}_{N}(\lambda)=\operatorname{det}\left(\lambda \mathbb{1}_{N}-J_{N}\right) .
$$

A direct calculation gives

$$
\begin{gathered}
\left(\lambda \mathbb{1}_{d N}-J_{d N}\right)^{-1}=\operatorname{diag}\left(B_{N}, \ldots, B_{N}\right), \quad B_{N}=\left(\lambda \mathbb{1}_{N}-J_{N}\right)^{-1}, \\
y^{*}(\vartheta)\left(\lambda \mathbb{1}_{d N}-J_{d N}\right)^{-1} y(\vartheta)=y_{1}^{*}\left(\vartheta_{1}\right) B_{N} y_{1}\left(\vartheta_{1}\right)+\ldots+y_{d}^{*}\left(\vartheta_{d}\right) B_{N} y_{d}\left(\vartheta_{d}\right), \\
y_{j}^{*}\left(\vartheta_{j}\right) B_{N} y_{j}\left(\vartheta_{j}\right)=\frac{1}{2^{N} \mathcal{D}_{N}(\lambda) d}\left(2^{N-1} \mathcal{D}_{N-1}(\lambda)+\cos \vartheta_{j}\right), \quad j \in \mathbb{N}_{d} .
\end{gathered}
$$

Substituting (6.19), (6.21) and (6.22) into the formula (6.18), we obtain

$$
\begin{array}{r}
\operatorname{det}\left(\lambda \mathbb{1}_{\nu}-J(\vartheta)\right)=\frac{\mathcal{D}_{N}^{d-1}(\lambda)}{2^{N}}\left(\lambda \widetilde{\mathcal{D}}_{N}(\lambda)-\widetilde{\mathcal{D}}_{N-1}(\lambda)-\frac{c_{0}}{d}\right) \\
\text { where } \widetilde{\mathcal{D}}_{n}(\lambda)=2^{n} \mathcal{D}_{n}(\lambda) .
\end{array}
$$

The expression $\widetilde{\mathcal{D}}_{n}(\lambda)$ satisfies the following recurrence relations (the Jacobi equation):

$$
\begin{gathered}
\widetilde{\mathcal{D}}_{n+1}(\lambda)=2 \lambda \widetilde{\mathcal{D}}_{n}(\lambda)-\widetilde{\mathcal{D}}_{n-1}(\lambda), \quad \forall n \in \mathbb{N}, \\
\text { where } \widetilde{\mathcal{D}}_{0}(\lambda)=1, \quad \widetilde{\mathcal{D}}_{1}(\lambda)=2 \lambda .
\end{gathered}
$$

Thus, $\widetilde{\mathcal{D}}_{n}(\lambda), n=1,2, \ldots$, are the Chebyshev polynomials of the second kind and the following identity holds

$$
\widetilde{\mathcal{D}}_{n}(\lambda)=\frac{\sin (n+1) \varphi}{\sin \varphi}, \quad \text { where } \lambda=\cos \varphi, \quad n \in \mathbb{N}
$$


Using the formulas (6.24) and (6.25), we rewrite (6.23) in the form

$$
\begin{aligned}
\operatorname{det}\left(\lambda \mathbb{1}_{\nu}-J(\vartheta)\right) & =\frac{\mathcal{D}_{N}^{d-1}(\lambda)}{2^{N}}\left(\frac{1}{2}\left(\widetilde{\mathcal{D}}_{N+1}(\lambda)-\widetilde{\mathcal{D}}_{N-1}(\lambda)\right)-\frac{c_{0}}{d}\right) \\
& =\frac{\mathcal{D}_{N}^{d-1}(\lambda)}{2^{N}}\left(\cos (N+1) \varphi-\frac{c_{0}}{d}\right) .
\end{aligned}
$$

Then the eigenvalues of the matrix $J(\vartheta)$ are solutions of the equations

$$
\mathcal{D}_{N}^{d-1}(\lambda)=0 \quad \text { or } \quad \cos (N+1) \varphi=\frac{c_{0}}{d} .
$$

From the first equation it follows that all flat bands $\mu_{n}, n=1, \ldots, N$, of the operator $J$ are defined by the formula (6.17). Then the set of all flat bands of the Laplacian $\Delta$ has the form (6.11). Since the range of the function $\frac{c_{0}}{d}$ is the segment $[-1,1]$, each $\lambda \in[-1,1]$ is a solution of the second equation in (6.27) for some $\vartheta \in \mathbb{T}^{d}$, i.e., an eigenvalue of the matrix $J(\vartheta)$. Thus, $\sigma_{a c}(J)=[-1,1]$. Consequently,

$$
\sigma_{a c}(\Delta)=[0,2]
$$

Proof of Theorem 2.4. Items $i)$ - iii) are direct consequences of Propositions 6.1 and 6.2.

\section{Crystal models}

It is known that the majority of common metals have either a face centered cubic (FCC) structure (Fig. 5), a body centered cubic (BCC) structure (Fig. 4), or a hexagonal close packed (HCP) structure (see [BM80]). The differences between these structures lead to different physical properties of bulk metals. For example, FCC metals, $\mathrm{Cu}, \mathrm{Au}, \mathrm{Ag}$, are usually soft and ductile, which means they can be bent and shaped easily. BCC metals are less ductile but stronger, for example iron, while HCP metals are usually brittle. Zinc is HCP and is difficult to bend without breaking, unlike copper.

We describe the spectrum of the Laplace and Schrödinger operators on the face centered and body centered cubic lattices.

7.1. Body-centered cubic lattice. The body-centered cubic lattice $\mathbf{B}_{2}$ is obtained from the cubic lattice $\mathbb{L}^{3}$ by adding one vertex in the center of each cube. This vertex is connected by an edge with each corner vertex of the cube (Fig. 4a). The fundamental graph of the lattice $\mathbf{B}_{2}$ consists of two vertices $v_{1}, v_{2}$ and 11 edges (Fig. $4 \mathrm{~b}$ ).

Proposition 7.1. i) The spectrum of the Laplacian on the body-centered cubic lattice $\mathbf{B}_{2}$ has the form

$$
\sigma(\Delta)=\sigma_{a c}(\Delta)=\sigma_{1}^{0} \cup \sigma_{2}^{0}=\left[0, \frac{11}{7}\right], \quad \sigma_{1}^{0}=[0,1], \quad \sigma_{2}^{0}=\left[1, \frac{11}{7}\right] .
$$

ii) Let the Schrödinger operator $H=\Delta+Q$ act on the body-centered cubic lattice $\mathbf{B}_{2}$, and let the potential $Q$ satisfy

$$
Q\left(v_{j}\right)=q_{j}, \quad j \in \mathbb{N}_{2}, \quad q_{2}=0 .
$$

Then the spectrum of the operator $H$ has the form

$$
\sigma(H)=\sigma_{a c}(H)=\sigma_{1} \cup \sigma_{2}=\left[\lambda_{1}^{-}, \lambda_{2}^{+}\right] \backslash \gamma_{1},
$$


(a)

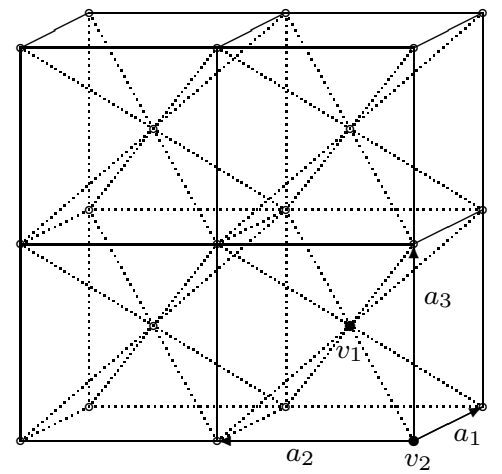

FiguRE 4. a) The body-centered cubic lattice $\mathbf{B}_{2}$; b) the fundamental graph. (b)

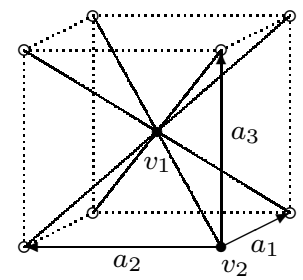

where

and the gap $\gamma_{1}$ is given by

$$
\begin{aligned}
& \lambda_{1}^{-}=\frac{11}{14}+\frac{1}{2} q_{1}-\frac{1}{2} \sqrt{\left(\frac{3}{7}+q_{1}\right)^{2}+\frac{16}{7}}, \\
& \lambda_{2}^{+}=\frac{11}{14}+\frac{1}{2} q_{1}+\frac{1}{2} \sqrt{\left(\frac{3}{7}+q_{1}\right)^{2}+\frac{16}{7}},
\end{aligned}
$$

$$
\gamma_{1}=\left(\lambda_{1}^{+}, \lambda_{2}^{-}\right)=\left\{\begin{array}{lll}
\left(\frac{10}{7}, 1+q_{1}\right), & \text { if } \quad \frac{3}{7}<q_{1} \\
\left(1+q_{1}, 1+q_{1}\right)=\varnothing, & \text { if } \quad-\frac{1}{7} \leqslant q_{1} \leqslant \frac{3}{7} . \\
\left(1+q_{1}, \frac{6}{7}\right), & \text { if } \quad q_{1}<-\frac{1}{7}
\end{array}\right.
$$

Proof. i) The fundamental graph $\Gamma_{*}$ of the body-centered cubic lattice $\mathbf{B}_{2}$ consists of two vertices $v_{1}, v_{2}$ with degrees $\varkappa_{1}=8, \varkappa_{2}=14 ; 11$ oriented edges

$$
\mathbf{e}_{1}=\mathbf{e}_{2}=\mathbf{e}_{3}=\left(v_{2}, v_{2}\right), \quad \mathbf{e}_{4}=\ldots=\mathbf{e}_{11}=\left(v_{1}, v_{2}\right)
$$

and their inverse edges (Fig. $4 b$ ). The indices of the fundamental graph edges are given by

$$
\begin{aligned}
& \tau\left(\mathbf{e}_{1}\right)=\tau\left(\mathbf{e}_{5}\right)=(1,0,0), \quad \tau\left(\mathbf{e}_{2}\right)=\tau\left(\mathbf{e}_{6}\right)=(0,1,0), \\
& \tau\left(\mathbf{e}_{3}\right)=\tau\left(\mathbf{e}_{7}\right)=(0,0,1), \quad \tau\left(\mathbf{e}_{4}\right)=(0,0,0), \\
& \tau\left(\mathbf{e}_{8}\right)=(1,1,0), \quad \tau\left(\mathbf{e}_{9}\right)=(1,0,1), \\
& \tau\left(\mathbf{e}_{10}\right)=(0,1,1), \quad \tau\left(\mathbf{e}_{11}\right)=(1,1,1) .
\end{aligned}
$$

For each $\vartheta=\left(\vartheta_{j}\right)_{j=1}^{3} \in \mathbb{T}^{3}$ the operator $\Delta(\vartheta)$ in the standard basis (4.1) has the form

$$
\begin{aligned}
& \Delta(\vartheta)=\left(\begin{array}{cc}
1 & \Delta_{12} \\
\bar{\Delta}_{12} & \Delta_{22}
\end{array}\right)(\vartheta), \\
& \left\{\begin{array}{l}
\Delta_{12}(\vartheta)=-\frac{1}{4 \sqrt{7}}\left(1+e^{-i \vartheta_{1}}\right)\left(1+e^{-i \vartheta_{2}}\right)\left(1+e^{-i \vartheta_{3}}\right) \\
\Delta_{22}(\vartheta)=1-\frac{c_{0}}{7}
\end{array}\right.
\end{aligned}
$$

where

$$
c_{0}=c_{1}+c_{2}+c_{3}, \quad c_{j}=\cos \vartheta_{j}, \quad j=1,2,3 .
$$


By a direct calculation, we get

$$
\operatorname{det}\left(\Delta(\vartheta)-\lambda \mathbb{1}_{2}\right)=(\lambda-1)^{2}+\frac{c_{0}}{7}(\lambda-1)-\frac{1}{14}\left(1+c_{1}\right)\left(1+c_{2}\right)\left(1+c_{3}\right) .
$$

The eigenvalues of each matrix $\Delta(\vartheta)$ are given by

$$
\lambda_{n}^{0}(\vartheta)=1-\frac{c_{0}}{14}+\frac{(-1)^{n}}{2} \sqrt{\frac{c_{0}^{2}}{49}+\frac{2}{7}\left(1+c_{1}\right)\left(1+c_{2}\right)\left(1+c_{3}\right)}, \quad n=1,2 .
$$

Thus, the spectrum of the Laplacian on the body-centered cubic lattice $\mathbf{B}_{2}$ has the form

$$
\begin{aligned}
& \sigma(\Delta)=\sigma_{a c}(\Delta)=\left[\lambda_{1}^{0-}, \lambda_{1}^{0+}\right] \cup\left[\lambda_{2}^{0-}, \lambda_{2}^{0+}\right], \\
& {\left[\lambda_{n}^{0-}, \lambda_{n}^{0+}\right]=\lambda_{n}^{0}\left(\mathbb{T}^{3}\right), \quad n=1,2 .}
\end{aligned}
$$

From the matrix property iii) it follows that

$$
\lambda_{1}^{0}(\vartheta) \leqslant 1 \leqslant \lambda_{2}^{0}(\vartheta), \quad \forall \vartheta \in \mathbb{T}^{3} .
$$

Investigating the function $\lambda_{2}^{0}$ for the maximum and using (2.1), (7.6), we obtain

$$
\begin{array}{ll}
\lambda_{1}^{0-}=\lambda_{1}^{0}(0)=0, & \lambda_{1}^{0+}=\lambda_{1}^{0}(\pi, \pi, \pi)=1, \\
\lambda_{2}^{0-}=\lambda_{2}^{0}(\pi, 0,0)=1, & \lambda_{2}^{0+}=\lambda_{2}^{0}(0,0,0)=\frac{11}{7} .
\end{array}
$$

ii) For each $\vartheta \in \mathbb{T}^{3}$ the operator $H(\vartheta)$ in the standard basis (4.1) has the form

$$
H(\vartheta)=\left(\begin{array}{cc}
1+q_{1} & \Delta_{12} \\
\bar{\Delta}_{12} & \Delta_{22}
\end{array}\right)(\vartheta)
$$

where $\Delta_{12}(\vartheta), \Delta_{22}(\vartheta)$ are given in (7.3) . By a direct calculation, we get

$$
\operatorname{det}\left(H(\vartheta)-\lambda \mathbb{1}_{2}\right)=(\lambda-1)^{2}+\left(\frac{c_{0}}{7}-q_{1}\right)(\lambda-1)-\frac{c_{0}}{7} q_{1}-\frac{1}{14}\left(1+c_{1}\right)\left(1+c_{2}\right)\left(1+c_{3}\right),
$$

where $c_{0}$ and $c_{j}, j=1,2,3$, are defined by (7.4). The eigenvalues of each matrix $H(\vartheta)$ are given by

$$
\lambda_{n}(\vartheta)=1-\frac{c_{0}}{14}+\frac{1}{2} q_{1}+\frac{(-1)^{n}}{2} \sqrt{\left(\frac{c_{0}}{7}+q_{1}\right)^{2}+\frac{2}{7}\left(1+c_{1}\right)\left(1+c_{2}\right)\left(1+c_{3}\right)}, \quad n=1,2 .
$$

By (2.1) and the matrix property iii), we have

$$
\begin{aligned}
& \lambda_{1}^{-}=\lambda_{1}(0)=\frac{11}{14}+\frac{1}{2} q_{1}-\frac{1}{2} \sqrt{\left(\frac{3}{7}+q_{1}\right)^{2}+\frac{16}{7}}, \\
& \lambda_{1}(\vartheta) \leqslant 1+q_{1} \leqslant \lambda_{2}(\vartheta), \quad \forall \vartheta \in \mathbb{T}^{3} .
\end{aligned}
$$

Then an investigation of the functions $\lambda_{1}, \lambda_{2}$ for the extremes gives

$$
\begin{aligned}
& \lambda_{1}^{+}=\lambda_{1}(\pi, \pi, \pi)=\left\{\begin{array}{rll}
1+q_{1}, & \text { if } & q_{1} \leqslant \frac{3}{7} \\
\frac{10}{7}, & \text { if } & q_{1}>\frac{3}{7}
\end{array},\right. \\
& \lambda_{2}^{-}=\lambda_{2}(\pi, 0,0)=\left\{\begin{array}{rll}
1+q_{1}, & \text { if } & q_{1} \geqslant-\frac{1}{7} \\
\frac{6}{7}, & \text { if } & q_{1}<-\frac{1}{7}
\end{array}\right. \text {, } \\
& \lambda_{2}^{+}=\lambda_{2}(0)=\frac{11}{14}+\frac{1}{2} q_{1}+\frac{1}{2} \sqrt{\left(\frac{3}{7}+q_{1}\right)^{2}+\frac{16}{7}} .
\end{aligned}
$$

This proves item ii). 
7.2. Face-centered cubic lattice. The face-centered cubic lattice $\mathbf{B}_{4}$ is obtained from the cubic lattice $\mathbb{L}^{3}$ by adding one vertex at the center of each cube face. This vertex is connected by an edge with each corner vertex of the cube face (Fig. $5 \mathrm{a}$ ). The fundamental graph of the lattice $\mathbf{B}_{4}$ consists of four vertices $v_{1}, v_{2}, v_{3}, v_{4}$ and 15 edges (Fig. [5b).

(a)

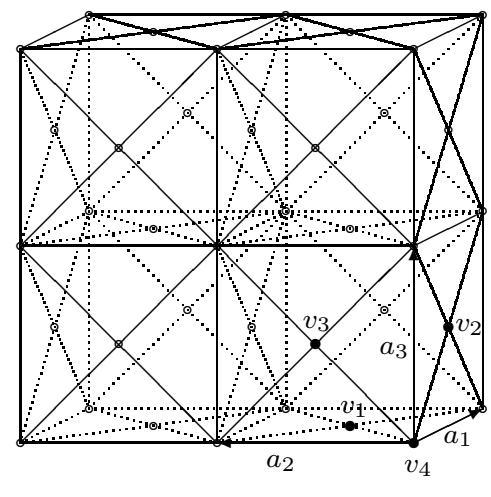

(b)

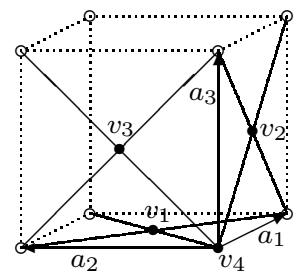

FiguRE 5. a) The face-centered cubic lattice; b) the fundamental graph.

Proposition 7.2. i) The spectrum of the Laplacian $\Delta$ on the face-centered cubic lattice $\mathbf{B}_{4}$ has the form

$$
\begin{aligned}
& \sigma(\Delta)=\sigma_{a c}(\Delta) \cup \sigma_{f b}(\Delta), \\
& \sigma_{a c}(\Delta)=[0,1] \cup\left[\frac{4}{3}, \frac{5}{3}\right], \quad \sigma_{f b}(\Delta)=\{1\},
\end{aligned}
$$

where the flat band $\{1\}$ has multiplicity 2 .

ii) Let the Schrödinger operator $H=\Delta+Q$ act on the face-centered cubic lattice $\mathbf{B}_{4}$, and let the potential $Q$ satisfy

$$
Q\left(v_{j}\right)=q_{j}, \quad j \in \mathbb{N}_{4}, \quad q_{4}=0 .
$$

Then the spectrum of the operator $H$ has the form

$$
\sigma(H)=\sigma_{a c}(H) \cup \sigma_{f b}(H)
$$

where

$$
\sigma_{f b}(H)= \begin{cases}\varnothing, & \text { if } q_{1}, q_{2}, q_{3} \text { are distinct (a generic potential); } \\ \left\{q_{(1)}+1\right\}, & \text { if } q_{j}=q_{k}=q_{(1)} \neq q_{n} \text { for some } j, k, n=1,2,3, \\ & n \neq j \neq k, \quad k \neq n \\ \left\{q_{(2)}+1\right\}, & \text { if } q_{1}=q_{2}=q_{3}=q_{(2)} .\end{cases}
$$

Moreover, the flat bands $\left\{q_{(1)}+1\right\}$ and $\left\{q_{(2)}+1\right\}$ have multiplicities 1 and 2 , respectively.

Proof. $i$ ) The fundamental graph $\Gamma_{*}$ of the face-centered cubic lattice $\mathbf{B}_{4}$ consists of four vertices $v_{1}, v_{2}, v_{3}, v_{4}$ with degrees $\varkappa_{1}=\varkappa_{2}=\varkappa_{3}=4, \varkappa_{4}=18 ; 15$ oriented edges

$$
\begin{aligned}
& \mathbf{e}_{1}=\mathbf{e}_{2}=\mathbf{e}_{3}=\left(v_{4}, v_{4}\right), \quad \mathbf{e}_{4}=\ldots=\mathbf{e}_{7}=\left(v_{1}, v_{4}\right), \\
& \mathbf{e}_{8}=\ldots=\mathbf{e}_{11}=\left(v_{2}, v_{4}\right), \quad \mathbf{e}_{12}=\ldots=\mathbf{e}_{15}=\left(v_{3}, v_{4}\right)
\end{aligned}
$$


and their inverse edges (Fig. 5b). The indices of the fundamental graph edges are given by:

$$
\begin{array}{llrl}
\tau\left(\mathbf{e}_{1}\right)=\tau\left(\mathbf{e}_{5}\right)=\tau\left(\mathbf{e}_{9}\right)=(1,0,0), & & \tau\left(\mathbf{e}_{7}\right)=(1,1,0), \\
\tau\left(\mathbf{e}_{2}\right)=\tau\left(\mathbf{e}_{6}\right)=\tau\left(\mathbf{e}_{13}\right)=(0,1,0), & & \tau\left(\mathbf{e}_{11}\right)=(1,0,1), \\
\tau\left(\mathbf{e}_{3}\right)=\tau\left(\mathbf{e}_{10}\right)=\tau\left(\mathbf{e}_{14}\right)=(0,0,1), & & \tau\left(\mathbf{e}_{15}\right)=(0,1,1) . \\
\tau\left(\mathbf{e}_{4}\right)=\tau\left(\mathbf{e}_{8}\right)=\tau\left(\mathbf{e}_{12}\right)=(0,0,0), &
\end{array}
$$

For each $\vartheta=\left(\vartheta_{j}\right)_{j=1}^{3} \in \mathbb{T}^{3}$ the operator $\Delta(\vartheta)$ in the standard basis (4.1) has the form

$$
\begin{gathered}
\Delta(\vartheta)=\left(\begin{array}{cccc}
1 & 0 & 0 & \Delta_{14} \\
0 & 1 & 0 & \Delta_{24} \\
0 & 0 & 1 & \Delta_{34} \\
\bar{\Delta}_{14} & \bar{\Delta}_{24} & \bar{\Delta}_{34} & \Delta_{44}
\end{array}\right)(\vartheta), \\
\left\{\begin{array}{l}
\Delta_{14}(\vartheta)=-\frac{1}{6 \sqrt{2}}\left(1+e^{-i \vartheta_{1}}\right)\left(1+e^{-i \vartheta_{2}}\right), \\
\Delta_{24}(\vartheta)=-\frac{1}{6 \sqrt{2}}\left(1+e^{-i \vartheta_{1}}\right)\left(1+e^{-i \vartheta_{3}}\right), \\
\Delta_{34}(\vartheta)=-\frac{1}{6 \sqrt{2}}\left(1+e^{-i \vartheta_{2}}\right)\left(1+e^{-i \vartheta_{3}}\right), \\
\Delta_{44}(\vartheta)=1-\frac{1}{9} c_{0},
\end{array}\right.
\end{gathered}
$$

where $c_{0}=c_{1}+c_{2}+c_{3}, c_{j}=\cos \vartheta_{j}, j=1,2,3$. By a direct calculation, we get

$$
\operatorname{det}\left(\Delta(\vartheta)-\lambda \mathbb{1}_{4}\right)=(\lambda-1)^{2}\left((\lambda-1)^{2}+\frac{c_{0}}{9}(\lambda-1)-\frac{1}{6}-\frac{c_{0}}{9}-\frac{1}{18} \eta\right)
$$

where

$$
\eta=\eta(\vartheta)=c_{1} c_{2}+c_{1} c_{3}+c_{2} c_{3} .
$$

From the matrix property iii) it follows that

$$
\lambda_{1}^{0}(\vartheta) \leqslant 1 \leqslant \lambda_{2}^{0}(\vartheta) \leqslant 1 \leqslant \lambda_{3}^{0}(\vartheta) \leqslant 1 \leqslant \lambda_{4}^{0}(\vartheta), \quad \forall \vartheta \in \mathbb{T}^{3} .
$$

Then the eigenvalues of the matrix $\Delta(\vartheta)$ are given by

$$
\begin{aligned}
\lambda_{1+3 s}^{0}(\vartheta) & =1-\frac{c_{0}}{18}+\frac{(-1)^{s+1}}{2} \sqrt{\left(\frac{c_{0}}{9}\right)^{2}+\frac{4 c_{0}}{9}+\frac{2}{3}+\frac{2}{9} \eta}, \\
s & =0,1, \quad \lambda_{2}^{0}(\vartheta)=\lambda_{3}^{0}(\vartheta)=1 .
\end{aligned}
$$

Thus, the spectrum of the Laplacian on the face-centered cubic lattice $\mathbf{B}_{4}$ has the form

$$
\begin{aligned}
& \sigma(\Delta)=\sigma_{a c}(\Delta) \cup \sigma_{f b}(\Delta), \\
& \sigma_{a c}(\Delta)=\left[\lambda_{1}^{0-}, \lambda_{1}^{0+}\right] \cup\left[\lambda_{4}^{0-}, \lambda_{4}^{0+}\right], \quad \sigma_{f b}(\Delta)=\{1\},
\end{aligned}
$$

where the flat band $\{1\}$ has multiplicity 2 and

$$
\left[\lambda_{n}^{0-}, \lambda_{n}^{0+}\right]=\lambda_{n}^{0}\left(\mathbb{T}^{3}\right), \quad n=1,4 .
$$

Next, using (2.1) and (7.12), we obtain

$$
\lambda_{1}^{0-}=\lambda_{1}^{0}(0)=0, \quad \lambda_{1}^{0+}=\lambda_{1}^{0}(\pi, \pi, \pi)=1 .
$$

A direct calculation gives

$$
\lambda_{4}^{0-}=\lambda_{4}^{0}(\pi, \pi, \pi)=\frac{4}{3}, \quad \lambda_{4}^{0+}=\lambda_{4}^{0}(0)=\frac{5}{3} .
$$


ii) For each $\vartheta \in \mathbb{T}^{3}$ the operator $H(\vartheta)$ has the form

$$
H(\vartheta)=\Delta(\vartheta)+q, \quad q=\operatorname{diag}\left(q_{1}, q_{2}, q_{3}, 0\right),
$$

where $\Delta(\vartheta)$ is defined by (7.11). We write the characteristic polynomial of the matrix $H(\vartheta)$ in the form of a linear combination of linearly independent functions:

$$
\begin{aligned}
\operatorname{det}\left(H(\vartheta)-\lambda \mathbb{1}_{4}\right) & =\eta_{1}(\lambda, q)+\eta_{2}(\lambda, q) c_{1}+\eta_{3}(\lambda, q) c_{2}+\eta_{4}(\lambda, q) c_{3} \\
& +\eta_{5}(\lambda, q) c_{1} c_{2}+\eta_{6}(\lambda, q) c_{1} c_{3}+\eta_{7}(\lambda, q) c_{2} c_{3},
\end{aligned}
$$

where $c_{j}=\cos \vartheta_{j}, j=1,2,3$,

$$
\begin{aligned}
\eta_{1}(\lambda, q)= & (\lambda-1)^{4}-\zeta_{1}(\lambda-1)^{3}+\left(\zeta_{2}-\frac{1}{6}\right)(\lambda-1)^{2}+\left(\frac{1}{9} \zeta_{1}-\zeta_{3}\right)(\lambda-1)-\frac{1}{18} \zeta_{2}, \\
\eta_{2}(\lambda, q)= & \frac{1}{9}(\lambda-1)^{3}-\frac{1}{9}\left(\zeta_{1}+1\right)(\lambda-1)^{2} \\
& +\left(\frac{1}{18} \zeta_{1}+\frac{1}{9} \zeta_{2}+\frac{1}{18} q_{3}\right)(\lambda-1)-\frac{1}{18} \zeta_{2}-\frac{1}{9} \zeta_{3}+\frac{1}{18} q_{1} q_{2}, \\
\eta_{3}(\lambda, q)= & \frac{1}{9}(\lambda-1)^{3}-\frac{1}{9}\left(\zeta_{1}+1\right)(\lambda-1)^{2} \\
& +\left(\frac{1}{18} \zeta_{1}+\frac{1}{9} \zeta_{2}+\frac{1}{18} q_{2}\right)(\lambda-1)-\frac{1}{18} \zeta_{2}-\frac{1}{9} \zeta_{3}+\frac{1}{18} q_{1} q_{3}, \\
\eta_{4}(\lambda, q)= & \frac{1}{9}(\lambda-1)^{3}-\frac{1}{9}\left(\zeta_{1}+1\right)(\lambda-1)^{2} \\
& +\left(\frac{1}{18} \zeta_{1}+\frac{1}{9} \zeta_{2}+\frac{1}{18} q_{1}\right)(\lambda-1)-\frac{1}{18} \zeta_{2}-\frac{1}{9} \zeta_{3}+\frac{1}{18} q_{2} q_{3}, \\
\eta_{5}(\lambda, q)= & -\frac{1}{18}(\lambda-1)^{2}+\frac{1}{18}\left(q_{2}+q_{3}\right)(\lambda-1)-\frac{1}{18} q_{2} q_{3}, \\
\eta_{6}(\lambda, q)= & -\frac{1}{18}(\lambda-1)^{2}+\frac{1}{18}\left(q_{1}+q_{3}\right)(\lambda-1)-\frac{1}{18} q_{1} q_{3}, \\
\eta_{7}(\lambda, q)= & -\frac{1}{18}(\lambda-1)^{2}+\frac{1}{18}\left(q_{1}+q_{2}\right)(\lambda-1)-\frac{1}{18} q_{1} q_{2}, \\
\zeta_{1}= & q_{1}+q_{2}+q_{3}, \\
\zeta_{2}= & q_{1} q_{2}+q_{1} q_{3}+q_{2} q_{3}, \\
\zeta_{3}= & q_{1} q_{2} q_{3} .
\end{aligned}
$$

A point $\lambda$ is a flat band of the operator $H$ if and only if

$$
\operatorname{det}\left(H(\vartheta)-\lambda \mathbb{1}_{4}\right)=0
$$

for all $\vartheta \in \mathbb{T}^{3}$. Since the linear combination (7.14) of linearly independent functions is equal to 0 , we obtain the system of equations

$$
\eta_{s}(\lambda, q)=0, \quad s \in \mathbb{N}_{7} .
$$

All solutions of this system have the form

$$
\lambda= \begin{cases}q_{(1)}+1, & \text { if } q_{j}=q_{k}=q_{(1)} \neq q_{n} \text { for some } j, k, n=1,2,3 \\ & n \neq j \neq k, \quad k \neq n \\ q_{(2)}+1, & \text { if } q_{1}=q_{2}=q_{3}=q_{(2)} .\end{cases}
$$


The roots $q_{(1)}+1$ and $q_{(2)}+1$ have multiplicities 1 and 2 , respectively. This proves item ii).

\section{Properties of matrices}

We denote by

$$
\lambda_{1}(A) \leqslant \ldots \leqslant \lambda_{\nu}(A)
$$

the eigenvalues of a self-adjoint $(\nu \times \nu)$-matrix $A$, arranged in non-decreasing order, counting multiplicities. The following well-known properties of matrices hold.

i) For each $n \in \mathbb{N}_{\nu}$ the eigenvalue $\lambda_{n}(A)$ satisfies the minimax principle:

$$
\begin{gathered}
\lambda_{n}(A)=\min _{S_{n} \subset \mathbb{C}^{\nu}} \max _{\substack{\|x\|=1 \\
x \in S_{n}}}\langle A x, x\rangle, \\
\lambda_{n}(A)=\max _{S_{\nu-n+1} \subset \mathbb{C}^{\nu}} \min _{\substack{\|x\|=1 \\
x \in S_{\nu-n+1}}}\langle A x, x\rangle,
\end{gathered}
$$

where $S_{n}$ denotes a subspace of dimension $n$ and the outer optimization is over all subspaces of the indicated dimension (see [HJ85, p. 180]).

ii) Let $A, B$ be self-adjoint $(\nu \times \nu)$-matrices. Then for each $n \in \mathbb{N}_{\nu}$ we have

$$
\lambda_{n}(A)+\lambda_{1}(B) \leqslant \lambda_{n}(A+B) \leqslant \lambda_{n}(A)+\lambda_{\nu}(B)
$$

(see [HJ85, Theorem 4.3.1]).

iii) Let $B=\left(\begin{array}{cc}A & y \\ y^{*} & a\end{array}\right)$ be a self-adjoint $(\nu+1) \times(\nu+1)$-matrix for some self-adjoint $(\nu \times \nu)$ matrix $A$, some real number $a$ and some vector $y \in \mathbb{C}^{\nu}$. Then

$$
\lambda_{1}(B) \leqslant \lambda_{1}(A) \leqslant \lambda_{2}(B) \leqslant \ldots \leqslant \lambda_{\nu}(B) \leqslant \lambda_{\nu}(A) \leqslant \lambda_{\nu+1}(B)
$$

(see [HJ85, Theorem 4.3.8]).

iv) Let $A=\left\{A_{j k}\right\}$ be a self-adjoint $(\nu \times \nu)$-matrix, and let $\Gamma(A)$ be a graph on $\nu$ vertices $v_{1}, \ldots, v_{\nu}$ such that there is an edge $\left(v_{j}, v_{k}\right)$ in it if and only if $A_{j k} \neq 0$. Then $A$ is irreducible if and only if the graph $\Gamma(A)$ is connected (see [HJ85, Theorem 6.2.24]).

$\mathrm{v})$ Let $A$ be an irreducible self-adjoint $(\nu \times \nu)$-matrix with nonnegative entries. Then the largest eigenvalue $\lambda=\|A\|$ of the matrix $A$ is simple, and for some vector $x$ with positive components we have $A x=\lambda x$ (see [HJ85, Theorem 8.4.4]).

vi) Let $M=\left(\begin{array}{ll}A & B \\ C & D\end{array}\right)$ be a $(\nu \times \nu)$-matrix for some square matrices $A, D$ and some matrices $B, C$. Then

$$
\operatorname{det} M=\operatorname{det} A \cdot \operatorname{det}\left(D-C A^{-1} B\right)
$$

(see [HJ85, pp. 21-22]).

vii) Let $V=\left\{V_{j k}\right\}$ be a self-adjoint $(\nu \times \nu)$-matrix, and let

$$
B=\operatorname{diag}\left\{B_{1}, \ldots, B_{\nu}\right\}, \quad B_{j}=\sum_{k=1}^{\nu}\left|V_{j k}\right| .
$$

Then the following estimates hold:

$$
-B \leqslant V \leqslant B
$$

(see, e.g., [K13]). 
Acknowledgments. Our study was supported by the RSF grant No. 18-11-00032.

\section{REFERENCES}

[A12] Ando K., Inverse scattering theory for discrete Schrödinger operators on the hexagonal lattice, Ann. Henri Poincaré 14 (2013), no. 2, 347-383.

[AIM16] Ando K., Isozaki H., Morioka H., Spectral properties of Schrödinger operators on perturbed lattices, Ann. Henri Poincare 17 (2016), no. 8, 2103-2171.

[A76] Ashcroft N. W., Mermin N. D., Solid state physics, Holt, Rinehart and Winston, New York, 1976.

[BM80] Barrett C. S., Massalski T. B., Structure of metals: crystallographic methods, principles and data, Pergamon Press, Oxford, 1980.

[BK13] Berkolaiko G., Kuchment P., Introduction to quantum graphs, Math. Surveys Monogr., v. 186, Amer. Math. Soc., Providence, RI, 2013.

[BS04] Birman M. Sh., Suslina T. A., Second order periodic differential operators. Threshold properties and homogenization, St. Petersburg Math. J. 15 (2004), no. 5, 639-714.

[BS99] Boutet de Monvel A., Sahbani J., On the spectral properties of discrete Schrödinger operators: (The multi-dimensional case), Rev. Math. Phys. 11 (1999), no. 9, 1061-1078.

[C97] Cattaneo C., The spectrum of the continuous Laplacian on a graph, Monatsh. Math. 124 (1997), no. 3, $215-235$.

[Ch97] Chung F., Spectral graph theory, CBMS Reg. Conf. Ser. Math., vol. 92, Amer. Math. Soc., Providence, RI, 1997.

[CDS95] Cvetkovic D., Doob M., Sachs H., Spectra of graphs. Theory and applications, Johann Ambrosius Barth, Heidelberg, 1995.

[GN98] Gerard C., Nier F., The Mourre theory for analytically fibered operators, J. Funct. Anal. 152 (1998), no. $1,202-219$.

[HKSW07] Harrison J. M., Kuchment P., Sobolev A., Winn B., On occurrence of spectral edges for periodic operators inside the Brillouin zone, J. Phys. A 40 (2007), no. 27, 7597-7618.

[HN09] Higuchi Y., Nomura Y., Spectral structure of the Laplacian on a covering graph, European J. Combin. 30 (2009), no. 2, 570-585.

[HJ85] Horn R, Johnson C., Matrix analysis, Cambridge Univ. Press, Cambridge, 1985.

[IK12] Isozaki H., Korotyaev E., Inverse problems, trace formulae for discrete Schrödinger operators, Ann. Henri Poincare 13 (2012), no. 4, 751-788.

[IM14] Isozaki H., Morioka H., A Rellich type theorem for discrete Schrödinger operators, Inverse Probl. Imaging 8 (2014), no. 2, 475-489.

[KiS87] Kirsch W., Simon S., Comparison theorem for gap of Schrödinger operators, J. Funct. Anal. 75 (1987), no. $2,396-410$.

[Ko10] Kopylova E. A., Dispersive estimates for discrete Schrödinger and Klein-Gordon equations, St. Petersburg Math. J. 21 (2010), no. 5, 743-760.

[K98] Korotyaev E., Estimates of periodic potentials in terms of gap lengths, Comm. Math. Phys. 197 (1998), no. $3,521-526$.

[K00] Korotyaev E., Estimates for the Hill operator. I, J. Differential Equations 162 (2000), no. 1, 1-26.

[K03] Korotyaev E., Characterization of the spectrum of Schrödinger operators with periodic distributions, Int. Math. Res. Not. 2003, no. 37, 2019-2031.

[K08] Korotyaev E., Effective masses for zigzag nanotubes in magnetic fields, Lett. Math. Phys. 83 (2008), no. $1,83-95$.

[K13] Korotyaev E., Stability estimates and inverse problems for block Jacobi matrices, preprint 2013.

[KM17] Korotyaev E., Moller J. S., Weighted estimates for the discrete Laplacian on the cubic lattice, preprint arXiv: 1701.03605

[KMR18] Korotyaev E., Moller J. S., Rasmussen M. G., Estimates for metric Laplacians on square lattice, preprint 2018.

[KS14] Korotyaev E., Saburova N., Schrödinger operators on periodic discrete graphs, J. Math. Anal. Appl. 420 (2014), no. 1, 576-611.

[KS15] Korotyaev E., Saburova N., Scattering on periodic metric graphs, preprint arXiv:1507.06441. 
[KS16] Korotyaev E., Saburova N., Estimates of bands for Laplacians on periodic equilateral metric graphs, Proc. Amer. Math. Soc. 144 (2016), no. 4, 1605-1617.

[KS16a] Korotyaev E., Saburova N., Effective masses for Laplacians on periodic graphs, J. Math. Anal. Appl. 436 (2016), no. 1, 104-130.

[KS18] Korotyaev E., Saburova N., Invariants for Laplacians on periodic graphs, preprint 2018.

[KS17a] Korotyaev E., Saburova N., Laplacians on periodic graphs with guides, J. Math. Anal. Appl. 455 (2017), no. 2, 1444-1469.

[KS17b] Korotyaev E., Saburova N., Schrödinger operators with guided potentials on periodic graphs, Proc. Amer. Math. Soc. 145 (2017), no. 11, 4869-4883.

[KoS99] Kostrykin V., Schrader R., Kirchhoffs rule for quantum wires, J. Phys. A 32 (1999), no. 4, 595-630.

[KSS98] Kotani M., Shirai T., Sunada T., Asymptotic behavior of the transition probability of a random walk on an infinite graph, J. Funct. Anal. 159 (1998), no. 2, 664-689.

[Ku89] Kuchment P., On the Floquet theory of periodic difference equations, Geometrical and Algebraical Aspects in Several Complex Variables (Cetraro, 1989), Sem. Conf., vol. 8, EditEl, Rende, 1991, pp. 201209.

[Ku02] Kuchment P., Graph models of wave propagation in thin structures, Waves Random Media 12 (2002), no. $4, \mathrm{R} 1-\mathrm{R} 24$.

[Ku14] Kutsenko A., Wave propagation through periodic lattice with defects, Comput. Mech. 54 (2014), no. 6, $1559-1568$.

[MW89] Mohar B., Woess W., A survey on spectra of infinite graphs, Bull. London Math. Soc. 21 (1989), no. 3, 209-234.

[PR18] Parra D., Richard S., Spectral and scattering theory for Schrödinger operators on perturbed topological crystals, Rev. Math. Phys. 30 (2018), no. 4, 185009.

[P12] Post O., Spectral analysis on graph-like spaces, Lecture Notes in Math., vol. 2039, Springer, Heidelberg, 2012.

[RR07] Rabinovich V. S., Roch S., Essential spectra of difference operators on $\mathbf{Z}^{n}$-periodic graphs, J. Phys. A 40 (2007), no. 33, 10109-10128.

[RS78] Reed M., Simon B., Methods of modern mathematical physics. IV, Analysis of operators, Acad. Press, New York, 1978.

[RS09] Rosenblum G., Solomjak M., On the spectral estimates for the Schrödinger operator on $\mathbb{Z}^{d}, d \geqslant 3$, Problems in Mathematical Analysis 41 (2009), 241-263.

[SS17] Sasaki I., Suzuki A., Essential spectrum of the discrete Laplacian on a perturbed periodic graph, J. Math. Anal. Appl. 446 (2017), no. 2, 1863-1881.

[SV01] Shaban W., Vainberg B., Radiation conditions for the difference Schrödinger operators, J. Appl. Anal. 80 (2001), no. 3-4, 525-556.

[Sk87] Skriganov M. M., Geometric and arithmetic methods in the spectral theory of multidimensional periodic operators, Proc. Steklov Inst. Math. 171 (1987), 1-121.

[S13] Sunada T., Topological crystallography, Surveys Tutorials Appl. Math. Sci., vol. 6, Springer, Tokyo, 2013.

[SS92] Sy P. W., Sunada T., Discrete Schrödinger operator on a graph, Nagoya Math. J. 125 (1992), 141-150.

[T73] Thomas L., Time dependent approach to scattering from impurities in a crystal, Comm. Math. Phys. 33 (1973), 335-343.

[Sh06] Shterenberg R., On the structure of the lower edge of the spectrum of the periodic magnetic Schrödinger operator with small magnetic potential, St. Petersburg Math. J. 17 (2006), no. 5, 865-873.

[V05] Veselic I., Spectral analysis of percolation Hamiltonians, Math. Ann. 331 (2005), no. 4, 841-865.

Department of Mathematical Analysis, Saint-Petersburg State University, UniversitetSkaya nab. 7/9, St. Petersburg, 199034, Russia, Korotyaev@gmail.com, E.Korotyaev@spbu.ru,

Department of Mathematical Analysis, Algebra and Geometry, Northern (Arctic) Federal University, Severnaya Dvina emb. 17, Arkhangelsk, 163002, Russia, N.saburova@gmail.com, N.SABUROVA@NARFU.RU 